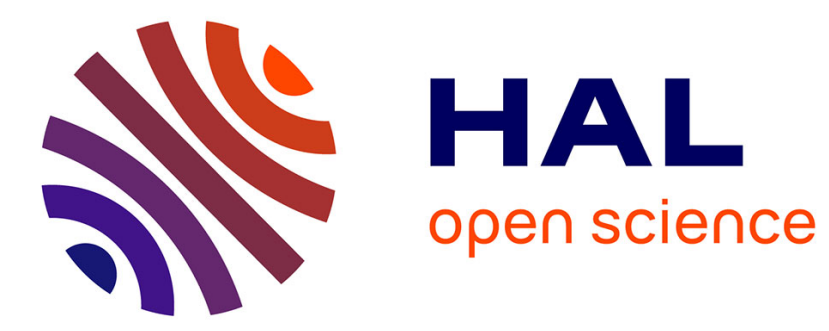

\title{
Numerical simulation of sheet metal forming using anisotropic strain-rate potentials
}

Meziane Rabahallah, Salima Bouvier, Tudor Balan, Brigitte Bacroix

\section{To cite this version:}

Meziane Rabahallah, Salima Bouvier, Tudor Balan, Brigitte Bacroix. Numerical simulation of sheet metal forming using anisotropic strain-rate potentials. Materials Science and Engineering: A, 2009, 517 (1-2), pp.261-275. 10.1016/j.msea.2009.03.078 . hal-01192743

\section{HAL Id: hal-01192743 \\ https://hal.science/hal-01192743}

Submitted on 3 Sep 2015

HAL is a multi-disciplinary open access archive for the deposit and dissemination of scientific research documents, whether they are published or not. The documents may come from teaching and research institutions in France or abroad, or from public or private research centers.
L'archive ouverte pluridisciplinaire HAL, est destinée au dépôt et à la diffusion de documents scientifiques de niveau recherche, publiés ou non, émanant des établissements d'enseignement et de recherche français ou étrangers, des laboratoires publics ou privés. 


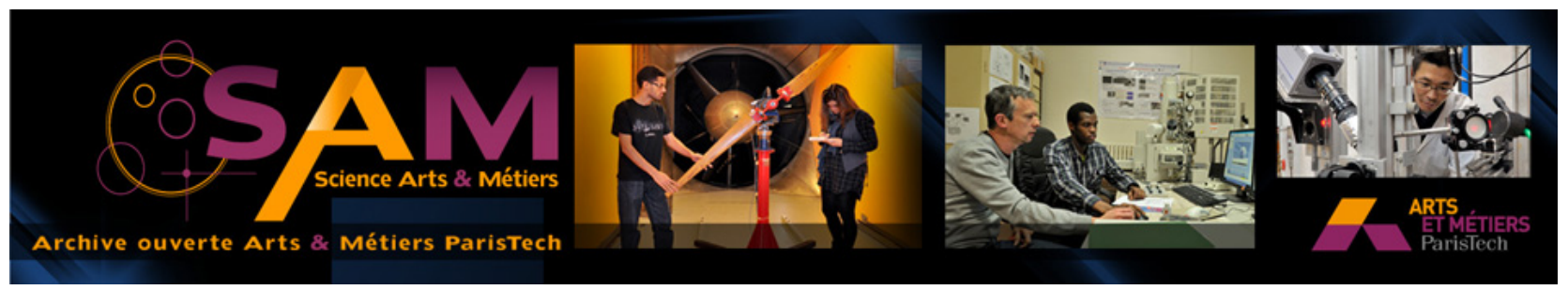

Science Arts \& Métiers (SAM)

is an open access repository that collects the work of Arts et Métiers ParisTech researchers and makes it freely available over the web where possible.

This is an author-deposited version published in: http://sam.ensam.eu

Handle ID: .http://hdl.handle.net/10985/9907

\section{To cite this version :}

Meziane RABAHALLAH, Salima BOUVIER, Tudor BALAN, Brigitte BACROIX - Numerical simulation of sheet metal forming using anisotropic strain-rate potentials - Materials Science \& Engineering A - Vol. 517, n¹-2, p.261-275 - 2009 


\title{
Numerical simulation of sheet metal forming using anisotropic strain-rate potentials
}

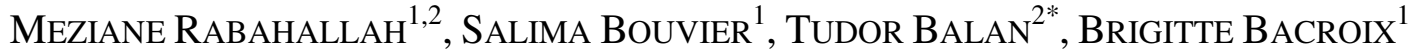 \\ ${ }^{1}$ LPMTM-CNRS, Université Paris 13, Institut Galilée, 99 rue J.-B. Clément, 93430 \\ Villetaneuse, France \\ ${ }^{2}$ LPMM, Arts et Métiers ParisTech-Metz Campus, 4 rue A. Fresnel, 57078 Metz Cedex 03, \\ France
}

\begin{abstract}
For numerical simulation of sheet metal forming, more and more advanced phenomenological functions are used to model the anisotropic yielding. The latter can be described by an adjustment of the coefficients of the yield function or the strain rate potential to the polycrystalline yield surface determined using crystal plasticity and X-ray measurements. Several strain rate potentials were examined by the present authors and compared in order to analyse their ability to model the anisotropic behaviour of materials using the methods described above to determine the material parameters. Following that, a specific elastic-plastic time integration scheme was developed and the strain rate potentials were implemented in the FE code. Comparison of the previously investigated potentials is continued in this paper in terms of numerical predictions of cup drawing, for different bcc and fcc materials. The identification procedure is shown to have an important impact on the accuracy of the FE predictions.
\end{abstract}

Keywords: sheet metal forming; anisotropy; finite element simulation; cup drawing; earing; strain-rate potential.

\footnotetext{
* Corresponding author. Tel.: +(33)3.87.37.54.60, Fax: +(33)3.87.37.54.70; E-mail address: tudor.balan@ensam.fr (T. Balan).
} 


\section{Introduction}

The role of numerical simulation in automotive sheet metal forming applications is continuously increasing, as new materials are considered in order to answer environmental and security issues. Plastic anisotropic was shown to have an important impact on the strain distribution during sheet forming as well as the formability of sheet materials. Thus, its accurate mathematical description, along with convenient parameter identification, is a key factor for realistic numerical simulations. This is particularly true when forming limits or springback must be predicted.

Phenomenological yield functions are commonly used do describe plastic anisotropy in finite element codes. Many such functions can be found in the literature (see e.g. reviews [1,2]). Usually associated with isotropic hardening for industrial applications, they can also be combined with kinematic or more advanced anisotropic hardening [3-7]. The simulation of cup drawing has been often used to address the accuracy of anisotropic yield criteria in FE simulations; Gotoh and Ishise [8] used Gotoh's yield criterion for simulations on a mild steel, Chung and Shah [9] applied the Yld91 criterion for a 2008-T4 aluminum alloy sheet, Andersson et al. [10] employed the criterion of Karafillis and Boyce for the limiting dome height (LDH) test, and Yoon et al. [11] implemented Yld2004-18p and demonstrated its ability to predict six- and eight-ear cup profiles.

Another approach to perform deep drawing simulations for anisotropic materials is to implement and apply computationally-efficient crystal plasticity models in finite element codes, e.g., [12-14]. This approach has the advantage of taking into account texture evolution and its associated deformation-induced anisotropy. However, this method remains very time consuming and has not been proven to provide better results when compared to the phenomenological approach.

Instead of an anisotropic yield function, a strain rate potential can be used in FE codes to simulate forming operations. Arminjon et al. [15,16] and Van Houtte et al. [17] proposed fourth-order and sixth-order strain rate functions, respectively. Barlat and Chung [18], Chung et al. [19], Barlat and Chung [20], and Kim et al. [21] introduced strain rate potentials that were pseudo-conjugate of the yield functions published earlier. Strain-rate potentials have proven very useful for inverse analysis and blank design applications [22-26]. Also, efficient rigid-plastic finite element implementations have been developed based on strain-rate potentials [27], allowing for large time increments and simple state update procedure. In order to predict unloading and springback phenomena, more expensive elastic-plastic finite element implementations of strain-rate potentials have been performed by Van Houtte et al. [28], Bacroix and Gilormini [29], Zhou et al. [30], and Li et al. [31]. Recently, Kim et al. [32] and Rabahallah et al. [33] proposed generic computer implementations for strain rate potentials (using shell and solid elements, respectively) and implemented several models, including the Srp2004-18p potential [21].

One of the most interesting features of strain rate potentials is related to the specific identification techniques based on texture $[15,17,34]$. In this framework, very flexible models have been proposed with a considerably large number of parameters that cannot be identified by classical methods $[35,36]$. The main drawback of this identification method is that it relies on the accuracy of the micromechanical model used to construct the reference data. 
Alternatively, the more classical identification method based on mechanical tests is currently applied to potentials (stress or strain-rate formulation) with numerous parameters (typically twenty) using a number of experimental points equal to the number of parameters. Given the large number of parameters, automatic identification is performed by minimization of an error function. On one hand, the experimental task becomes more and more laborious and errorprone. On the other hand, until now, the impact of the identification technique on the finite element results has not been investigated.

In addition to the efforts devoted to the prediction of the earing profile during cup drawing using FE simulations, there have been some analytical attempts at this prediction. The main difficulty in analytical approaches is determination of the major mechanical parameters that are directly related to the earing profile. Bourne and Hill [37] observed that the angular positions, $\alpha$, of the ears of various mild steel sheets correspond to the maxima of $r(\pi / 2-\alpha)$ and to the minima of $\sigma(\pi / 2-\alpha)$. Hosford and Caddell [38] and Chung et al. [24] provided a quantitative trend between $r$-value anisotropy and possible earing profiles in a mild steel and an aluminum alloy, respectively. In a different approach, Barlat et al. [22,39] attempted to correlate the yield stress anisotropy (not $r$-values) to the earing trends. More quantitative evaluations of the cup heights have been proposed by Yoon et al. [11], based on an analytical approach considering the $r$-value directionality as a main contributor to the earing profile derived from the work of Chung et al. [24].

The aim of this paper is to evaluate the finite element predictions of several strain rate potentials, in particular the recent Srp2004-18p model. Five different b.c.c. and f.c.c. sheet materials that exhibit strong dissimilar initial anisotropy have been selected for this purpose. Particular attention is given to the impact of the parameter identification method on the FE predictions. For this purpose, Srp2004-18p has been implemented in the FE code Abaqus, together with other models used in the comparison [33]. The modeling and computational issues of this work are summarized in Section 2, and the FE simulations are analyzed in Section 3, which is the main part of the paper.

\section{Constitutive modeling, computer implementation, and parameter identification}

This section summarizes the theoretical modeling and numerical methodologies used for the numerical simulations. For more details, the reader may refer to Kim et al. [21] and Rabahallah et al. [36] for strain-rate potential models, Rabahallah et al. [33] for the complete elastic-plasticity model and its numerical implementation, and to Rabahallah et al. [34] for the parameter identification of the strain-rate potentials.

\subsection{Strain-rate-potential-based elastic-plasticity model}

In order to represent the rate-insensitive plastic behavior of materials phenomenologically, it is typical to use a yield function, a flow rule, and a hardening law. The yield function $\Phi$ gives the stress level at which yielding occurs for a given stress mode, and its gradient (the normal to the yield surface at the loading point) gives the direction of the plastic strain rate $\mathbf{D}^{p}$, while 
the plastic multiplier $\dot{\lambda}$ defines its intensity. Alternatively, a dual strain rate potential $\Psi$ $[40,41]$ can be associated with any convex stress potential (or yield surface):

$$
\Psi\left(\mathbf{D}^{p}\right)=\dot{\lambda}
$$

which is expressed as a function of the traceless plastic strain rate tensor, while its gradient with respect to $\mathbf{D}^{p}$ leads to the direction of the stress deviator $\boldsymbol{\sigma}^{\prime}$; i.e.,

$$
\boldsymbol{\sigma}^{\prime}=\tau \frac{\partial \Psi}{\partial \mathbf{D}^{p}}
$$

In Eq. (2), $\tau$ is a proportionality factor necessary to scale the stress deviator. Its value is related to the reference stress, such as the uniaxial stress in the rolling direction, or to the crystallographic critical shear stress. When kinematic hardening is taken into account in the modeling, Eq. (2) takes the more general form:

$$
\boldsymbol{\sigma}^{\prime}=\mathbf{X}+\tau \frac{\partial \Psi}{\partial \mathbf{D}^{p}}
$$

where $\mathbf{X}$ is the second order backstress tensor used to describe the translation of the yield surface in the stress space. In addition to the plastic potential definition and the flow rule, the hypo-elastic response of the material is modeled by:

$$
\dot{\boldsymbol{\sigma}}=\mathbf{C}^{e}:\left(\mathbf{D}-\mathbf{D}^{p}\right)
$$

where $\dot{\boldsymbol{\sigma}}$ is the rate of the Cauchy stress tensor $\boldsymbol{\sigma}, \mathbf{D}$ is the total strain rate tensor, and $\mathbf{C}^{e}$ is the fourth-order elasticity tensor. A classical nonlinear hardening model is considered, involving both isotropic and kinematic hardening:

$$
\begin{aligned}
& \tau=\tau_{0}+R \\
& \dot{R}=C_{\mathrm{R}}\left(R_{\text {sat }}-R\right) \dot{\lambda}, \quad R(0)=0 ; \\
& \dot{\mathbf{X}}=C_{\mathrm{x}}\left(X_{\mathrm{sat}} \frac{\partial \Psi}{\partial \mathbf{D}^{p}}-\mathbf{X}\right) \dot{\lambda}, \quad \mathbf{X}(0)=\mathbf{0} .
\end{aligned}
$$

where $\tau_{0}, C_{\mathrm{R}}, R_{\mathrm{sat}}, C_{\mathrm{x}}$ and $X_{\text {sat }}$ are material parameters.

\subsection{Plastic potentials considered in this work}

Five strain-rate potentials are compared in this work by means of several f.c.c. and b.c.c. sheet materials. The von Mises and Hill potentials are derived as the analytical duals of the corresponding widely known yield functions. The fourth-order potential Quartus $[15,16]$ can be considered as conjugate to the three-dimension extension of the fourth-order yield function given by Gotoh [42]. Finally, two strain-rate potentials with noninteger exponents are considered, as proposed by Barlat and co-workers: Srp93 [18] and Srp2004-18p [20,21].

The quadratic strain-rate potential can be written as: 


$$
\Psi\left(\mathbf{D}^{p}\right)=\left[\frac{4 F}{\Delta}\left(D_{11}^{p}\right)^{2}+\frac{4 G}{\Delta}\left(D_{22}^{p}\right)^{2}+\frac{4 H}{\Delta}\left(D_{33}^{p}\right)^{2}+\frac{2}{L}\left(D_{23}^{p}\right)^{2}+\frac{2}{M}\left(D_{31}^{p}\right)^{2}+\frac{2}{N}\left(D_{12}^{p}\right)^{2}\right]^{\frac{1}{2}}
$$

where $\Delta=F H+F G+H G$ and the three axes 1, 2, and 3 are the rolling, transverse, and normal directions, respectively, in the case of rolled orthotropic sheet metal. For an isotropic material, the von Mises potential is recovered by setting the Hill's parameter values to $F=G=H=1 / 2$ and $L=M=N=3 / 2$.

The fourth-order Quartus potential reads [15]:

$$
\psi\left(\mathbf{D}^{p}\right)=\sum_{k=1}^{22} \alpha_{k} \frac{X_{k}\left(\mathbf{D}^{p}\right)}{\left\|\mathbf{D}^{p}\right\|^{3}}
$$

where $\alpha_{k}, k=1, \ldots, 22$ are material (anisotropy) parameters and $X_{k}$ are 22 terms depending on the plastic strain rate tensor components:

$$
\begin{array}{ccc}
X_{1}=\left(D_{11}^{p}\right)^{4} & X_{2}=\left(D_{22}^{p}\right)^{4} & X_{3}=\left(D_{23}^{p}\right)^{4} \\
X_{4}=\left(D_{13}^{p}\right)^{4} & X_{5}=\left(D_{12}^{p}\right)^{4} & X_{6}=\left(D_{11}^{p}\right)^{3} D_{22}^{p} \\
X_{7}=\left(D_{22}^{p}\right)^{3} D_{11}^{p} & X_{8}=\left(D_{11}^{p}\right)^{2}\left(D_{22}^{p}\right)^{2} & X_{9}=\left(D_{11}^{p}\right)^{2}\left(D_{23}^{p}\right)^{2} \\
X_{10}=\left(D_{11}^{p}\right)^{2}\left(D_{13}^{p}\right)^{2} & X_{11}=\left(D_{11}^{p}\right)^{2}\left(D_{12}^{p}\right)^{2} & X_{12}=\left(D_{22}^{p}\right)^{2}\left(D_{23}^{p}\right)^{2} \\
X_{13}=\left(D_{22}^{p}\right)^{2}\left(D_{13}^{p}\right)^{2} & X_{14}=\left(D_{22}^{p}\right)^{2}\left(D_{12}^{p}\right)^{2} & X_{15}=\left(D_{23}^{p}\right)^{2}\left(D_{13}^{p}\right)^{2} \\
X_{16}=\left(D_{23}^{p}\right)^{2}\left(D_{12}^{p}\right)^{2} & X_{17}=\left(D_{13}^{p}\right)^{2}\left(D_{12}^{p}\right)^{2} & X_{18}=D_{11}^{p} D_{22}^{p}\left(D_{23}^{p}\right)^{2} \\
X_{19}=D_{11}^{p} D_{22}^{p}\left(D_{13}^{p}\right)^{2} & X_{20}=D_{11}^{p} D_{22}^{p}\left(D_{12}^{p}\right)^{2} & X_{21}=D_{11}^{p} D_{23}^{p} D_{13}^{p} D_{12}^{p} \\
& X_{22}=D_{22}^{p} D_{23}^{p} D_{13}^{p} D_{12}^{p} &
\end{array}
$$

Finally, the Srp2004-18p potential takes the mathematical form [21]:

$$
\Psi\left(\mathbf{D}^{p}\right)=\left[\frac{1}{2^{2-b}+2}\left(\left|\tilde{\mathbf{E}}_{1}^{\prime}\right|^{b}+\left|\tilde{\mathbf{E}}_{2}^{\prime}\right|^{b}+\left|\tilde{\mathbf{E}}_{3}^{\prime}\right|^{b}+\left|\tilde{\mathbf{E}}_{2}^{\prime \prime}+\tilde{\mathbf{E}}_{3}^{\prime \prime}\right|^{b}+\left|\tilde{\mathbf{E}}_{3}^{\prime \prime}+\tilde{\mathbf{E}}_{1}^{\prime \prime}\right|^{b}+\left|\tilde{\mathbf{E}}_{1}^{\prime \prime}+\tilde{\mathbf{E}}_{2}^{\prime \prime}\right|^{b}\right)\right]^{\frac{1}{b}},
$$

where $\left[\tilde{E}_{i}^{\prime}\right]$ and $\left[\tilde{E}_{i}^{\prime \prime}\right]$ are the principal values of $\tilde{\mathbf{D}}^{p^{\prime}}$ and $\tilde{\mathbf{D}}^{p^{\prime \prime}}$, defined respectively by the two linear transformations hereafter:

$$
\begin{aligned}
& \tilde{\mathbf{D}}^{p^{\prime}}=\mathbf{A}^{\prime} \cdot \mathbf{I}_{s 4}^{\prime} \cdot \hat{\mathbf{D}}^{p}, \\
& \tilde{\mathbf{D}}^{p^{\prime \prime}}=\mathbf{A}^{\prime \prime} \cdot \mathbf{I}_{s 4}^{\prime} \cdot \hat{\mathbf{D}}^{p} .
\end{aligned}
$$

In Eqs. (12) and (13), $\mathbf{I}_{s 4}^{\prime}$ designates the unit tensor in the space of fourth-order deviatoric and symmetric tensors while the fourth-order arrays $\mathbf{A}^{\prime}$ and $\mathbf{A}^{\prime \prime}$ contain anisotropy coefficients. For the case of orthotropic symmetry, they can be represented as the following $6 \times 6$ arrays: 


$$
\mathbf{A}^{\prime}=\left[\begin{array}{cccccc}
0 & -a_{1} & -a_{2} & 0 & 0 & 0 \\
-a_{3} & 0 & -a_{4} & 0 & 0 & 0 \\
-a_{5} & -a_{6} & 0 & 0 & 0 & 0 \\
0 & 0 & 0 & a_{7} & 0 & 0 \\
0 & 0 & 0 & 0 & a_{8} & 0 \\
0 & 0 & 0 & 0 & 0 & a_{9}
\end{array}\right] \text { and } \mathbf{A}^{\prime \prime}=\left[\begin{array}{cccccc}
0 & -a_{10} & -a_{11} & 0 & 0 & 0 \\
-a_{12} & 0 & -a_{13} & 0 & 0 & 0 \\
-a_{14} & -a_{15} & 0 & 0 & 0 & 0 \\
0 & 0 & 0 & a_{16} & 0 & 0 \\
0 & 0 & 0 & 0 & a_{17} & 0 \\
0 & 0 & 0 & 0 & 0 & a_{18}
\end{array}\right]
$$

where $a_{i}$ are the material parameters. In order to use these compact notations, the $\mathbf{D}^{p}$-like tensors are written here as 6-component vectors; e.g., $\mathbf{D}^{p}=\left[\begin{array}{lllllll}D_{11}^{p} & D_{22}^{p} & D_{33}^{p} & D_{23}^{p} & D_{31}^{p} & D_{12}^{p}\end{array}\right]^{T}$, with components in the frame of material symmetry. The isotropic case is obtained for $a_{1}=a_{2}=\ldots=a_{18}=1$ and $b=4 / 3$ or $3 / 2$ for b.c.c. or f.c.c. materials, respectively.

The Srp93 potential [18] can be recovered from the previous model by enforcing

which is to say:

$$
\mathbf{A}^{\prime} \cdot \mathbf{I}_{s 4}^{\prime}=\mathbf{A}^{\prime \prime} \cdot \mathbf{I}_{s 4}^{\prime}=\mathbf{A},
$$

$$
\left\{\begin{array} { l } 
{ a _ { 1 } = a _ { 1 0 } = \frac { 1 } { 3 } ( 2 \cdot a _ { 3 } ^ { \prime } + a _ { 2 } ^ { \prime } ) } \\
{ a _ { 2 } = a _ { 1 1 } = \frac { 1 } { 3 } ( 2 \cdot a _ { 2 } ^ { \prime } + a _ { 3 } ^ { \prime } ) } \\
{ a _ { 3 } = a _ { 1 2 } = \frac { 1 } { 3 } ( 2 \cdot a _ { 3 } ^ { \prime } + a _ { 1 } ^ { \prime } ) }
\end{array} \quad \left\{\begin{array} { l } 
{ a _ { 4 } = a _ { 1 3 } = \frac { 1 } { 3 } ( 2 \cdot a _ { 1 } ^ { \prime } + a _ { 3 } ^ { \prime } ) } \\
{ a _ { 5 } = a _ { 1 4 } = \frac { 1 } { 3 } ( 2 \cdot a _ { 2 } ^ { \prime } + a _ { 1 } ^ { \prime } ) } \\
{ a _ { 6 } = a _ { 1 5 } = \frac { 1 } { 3 } ( 2 \cdot a _ { 1 } ^ { \prime } + a _ { 2 } ^ { \prime } ) }
\end{array} \quad \left\{\begin{array}{l}
a_{7}=a_{16}=a_{4}^{\prime} \\
a_{8}=a_{17}=a_{5}^{\prime} \\
a_{9}=a_{18}=a_{6}^{\prime}
\end{array}\right.\right.\right.
$$

Recently, a generalization of Srp93 and Srp2004-18p has been proposed by Rabahallah et al. [36], involving an arbitrary number of linear transformations.

\subsection{Computer implementation}

An implicit backward Euler state update algorithm has been developed for the aforementioned elastic-plastic model in the framework of finite strains; this algorithm is described in detail in [33]. The flow rule (3) and of the deviatoric part of the hypo-elastic law (4) take the following discrete (incremental) forms

$$
\begin{gathered}
\boldsymbol{\sigma}_{n+1}^{\prime}=\mathbf{X}_{n+1}+\tau_{n+1} \frac{\partial \Psi}{\partial\left(\Delta \boldsymbol{\varepsilon}^{p}\right)} . \\
\boldsymbol{\sigma}_{n+1}^{\prime}=\boldsymbol{\sigma}_{n}^{\prime}+\mathbf{C}^{e}:\left(\Delta \boldsymbol{\varepsilon}^{\prime}-\Delta \boldsymbol{\varepsilon}^{p}\right),
\end{gathered}
$$

where $\mathbf{X}_{n+1}$ and $\tau_{n+1}$ are the internal variables at the end of the increment, obtained as functions of the plastic strain increment $\Delta \boldsymbol{\varepsilon}^{p}$ by time integration of equations (5)-(7); $\boldsymbol{\sigma}_{n}^{\prime}$ and $\boldsymbol{\sigma}_{n+1}^{\prime}$ denote the deviatoric stress at the beginning and at the end of the time increment, respectively, while $\Delta \boldsymbol{\varepsilon}^{\prime}$ is the (given) deviatoric strain increment. By eliminating $\boldsymbol{\sigma}_{n+1}^{\prime}$ from 
these two equations, the following nonlinear equation is obtained, with the traceless plastic strain rate increment $\Delta \boldsymbol{\varepsilon}^{p}$ as the main unknown:

$$
\mathbf{X}_{n+1}\left(\Delta \boldsymbol{\varepsilon}^{p}\right)+\tau_{n+1}\left(\Delta \boldsymbol{\varepsilon}^{p}\right) \frac{\partial \Psi\left(\Delta \boldsymbol{\varepsilon}^{p}\right)}{\partial\left(\Delta \boldsymbol{\varepsilon}^{p}\right)}-\mathbf{C}^{e}:\left(\Delta \boldsymbol{\varepsilon}^{\prime}-\Delta \boldsymbol{\varepsilon}^{p}\right)-\boldsymbol{\sigma}_{n}^{\prime}=\mathbf{0}
$$

The resolution of this nonlinear system is performed with a Newton-Raphson method. A particularity of the strain rate potential approach is the lack of an explicit yield condition, which allows one to decide whether or not the elastic trial violates the yield surface. This problem is overcome by solving a minimization problem proposed by Bacroix and Gilormini [29], which states that for a given stress tensor $\boldsymbol{\sigma}$

$$
\underset{\mathbf{N}}{\operatorname{Min}}\left[\tau \Psi(\mathbf{N})-\left(\boldsymbol{\sigma}^{\prime}-\mathbf{X}\right): \mathbf{N}\right]\left\{\begin{array}{cccc}
<0 & \text { if } & \boldsymbol{\sigma} & \text { lays outside the yield surface } \\
=0 & \text { if } & \boldsymbol{\sigma} & \text { lays on the yield surface } \\
>0 & \text { if } & \boldsymbol{\sigma} & \text { lays inside the yield surface. }
\end{array}\right.
$$

where $\mathbf{N}$ is a unit-length deviatoric tensor designating the plastic strain rate direction. In summary, the constitutive algorithm makes use of two nonlinear numerical procedures, namely the resolution of the nonlinear system and of the minimization problem for the yield condition. These procedures may diverge in case of too large strain increments, especially when highly nonlinear anisotropic potentials are used. In order to avoid early divergence in the constitutive algorithm, a sub-stepping technique has also been developed and implemented. This is an extension to strain-rate potentials of the sub-stepping procedure introduced by Yoon et al. [11,43] and it is described in detail in [33].

The resulting constitutive algorithm has been implemented in Abaqus/Standard and has been used to perform all the numerical simulations presented in the current paper. Several numerical validations $[33,44]$ have shown that this implementation of the strain-rate-potentialbased approach induces no extra computing time as compared to the Abaqus built-in update algorithm that makes use of the yield-function approach.

\subsection{Parameter identification}

Parameter identification is an important issue, especially when a large number of parameters must be determined. The advanced plastic potentials adopted in this work require about twenty parameters (18 and 22 for the Srp2004-18p and Quartus potentials, respectively). Classically, the parameter identification makes use of material data issued from mechanical tests, such as yield stress values and/or $r$-values from tensile tests, biaxial tests, shear tests, plane strain tests, etc. An alternative method uses a micromechanical model and crystallographic texture data for the identification and has been extensively used for the parameter identification of plastic potentials $[12,15,17,34,45]$. Both identification techniques have been used in this work, and the impact of the identification procedure on the results of the FE simulations is one of its main objectives. The two identification methods are briefly discussed hereafter; the reader should refer to $[34,44]$ for more details on the identification procedure and for a detailed presentation of the parameter identification results and comparison. 


\subsubsection{Parameter identification using experimental mechanical tests}

The experimental data considered for identification is a combination of in-plane uniaxial tensile strength and $r$ values along various directions, as well as the balanced biaxial strength $\sigma_{b}\left(=\sigma_{11}=\sigma_{22}\right)$ and strain rate ratio $\mathrm{r}_{b}\left(=D_{22}^{p} / D_{11}^{p}\right)$. Whether the number of experimental data is equal to or larger than the number of coefficients of the potential considered, it is necessary to apply the least-squares method based on an objective function for the identification. Outof-plane property data, such as pure shear or uniaxial tension at $45^{\circ}$ from symmetry axes, were assumed to be isotropic in this work in order to calculate the out-of-plane anisotropy coefficients. However, more generally, any other convenient deformation state could be considered for the out-of plane properties. When all of the input data are selected, the coefficients are obtained by minimizing the following objective function (see [21]):

$$
F_{\text {Mech }}=\frac{\tau^{2}}{\bar{\sigma}^{2}}\left\{\begin{array}{c}
\sum_{m}\left[w_{m 1}\left(\frac{\partial \psi}{\partial \varepsilon_{11}^{m}}-\frac{\partial \psi}{\partial \varepsilon_{33}^{m}}-\frac{\sigma_{11}^{m}}{\tau}\right)^{2}+w_{m 2}\left(\frac{\partial \psi}{\partial \varepsilon_{22}^{m}}-\frac{\partial \psi}{\partial \varepsilon_{33}^{m}}\right)^{2}\right]+ \\
w_{r 1}\left(\frac{\partial \psi}{\partial \varepsilon_{11}}-\frac{\partial \psi}{\partial \varepsilon_{33}}-\frac{\sigma_{b}}{\tau}\right)^{2}+w_{r 2}\left(\frac{\partial \psi}{\partial \varepsilon_{11}^{m}}-\frac{\partial \psi}{\partial \varepsilon_{22}^{m}}\right)^{2}+\sum_{n} w_{n}\left(\frac{\partial \psi}{\partial \varepsilon_{i j}^{n}}-\frac{\tau_{i j}^{n}}{\tau}\right)^{2}
\end{array}\right\}
$$

Here, $m$ represents the number of uniaxial yield stresses and $r$ values available. The first term under the first summation sign corresponds to the (arbitrary) longitudinal uniaxial tensile stress (direction 1) when the imposed strain rate state is calculated with the associated $r$ value. The second term under the first summation sign corresponds to the (vanishing) stress transverse (direction 2) to the previously calculated longitudinal direction. The third and fourth terms correspond to balanced biaxial stress conditions when the imposed strain rate state is calculated with the associated $r_{b}$ value. Finally, $n$ represents the number of experimental pure shear yield stresses available (from out of plane properties in this work). Each term in the objective function is multiplied by a weight $w$.

In Eq.(20), the potential is defined with respect to the strain components instead of the strain rate components, since the potential can be redefined simply by replacing the strain rate with true (or logarithmic) strain when the deformation is monotonously proportional [46].

\subsubsection{Texture-based parameter identification using a micromechanical model}

For any given plastic strain rate direction $\mathbf{N}$, the micromechanical Taylor-Bishop-Hill model $(\mathrm{TBH})$ is used to calculate the normalized plastic work rate $\Pi_{T B H}^{P}(\mathbf{N})=\dot{W}_{T B H}^{P}(\mathbf{N}) / \tau_{c}=\left(\boldsymbol{\sigma}^{\prime} / \tau_{c}\right): \mathbf{N}$, where $\dot{W}_{T B H}^{P}(\mathbf{N})$ is the plastic work rate and $\tau_{c}$ is the crystallographic critical shear stress. This quantity corresponds to the plastic potential since it can be easily shown (see [34]) that, in the framework of the adopted phenomenological modelling,

$$
\psi(\mathbf{N})=\frac{\dot{W}^{p}(\mathbf{N})}{\tau} .
$$


In other words, for any strain rate direction $\mathbf{N}_{i}$, the functions $\Pi_{T B H}^{P}\left(\mathbf{N}_{i}\right)$ and $\psi\left(\mathbf{N}_{i}\right)$ correspond to the plastic power associated to a unit-norm strain rate tensor and normalized by $\tau_{c}$. The coefficients of the plastic potential $\psi$ are thus identified by minimizing the objective function:

$$
F_{T e x}=\frac{\sum_{i} w_{i}\left[\Pi_{T B H}^{P}\left(\mathbf{N}_{i}\right)-\psi\left(\mathbf{N}_{i}\right)\right]^{2}}{\sum_{i}\left[\Pi_{T B H}^{P}\left(\mathbf{N}_{i}\right)\right]^{2}},
$$

with respect to the coefficients of the chosen potential. The sum is performed over 80000 selected 3D strain rate directions [15]. Then, the micromechanical values $\Pi_{T B H}^{P}\left(\mathbf{N}_{i}\right)$ are computed for all of these directions. This is a lengthy task, but has to be performed only once for a given initial crystallographic texture.

The nonlinear least-squares-problem is solved using a Levenberg-Marquardt minimization algorithm [47]. This algorithm requires the calculation of the objective function and its firstorder derivatives with respect to the parameters subject to identification.

\section{Finite element simulation of cup drawing processes}

Cup drawing is a simple and typical sheet forming process, which is widely used to illustrate sheet metal anisotropy and to evaluate the prediction capabilities of yield functions. Although more complex sheet metal forming processes can be simulated with the existing implementation [33], cylindrical cup drawing is adopted here for several reasons:

- This test has been widely used to evaluate different materials and corresponding data is available in the literature;

- Sheet metal providers systematically perform cup drawing tests for all sheet materials;

- Although simple, this test is relevant for industrial forming of beverage cans and other packaging products. It also has practical relevance for more complex sheet forming processes, especially in terms of in-plane anisotropy;

- Cup drawing involves large strains, together with contact evolution, thus requiring a complete numerical simulation;

- Most importantly, the cup profile has been shown to be sensitive to the initial anisotropy (and to the corresponding models and parameters) and to illustrate it in a very intuitive way. On the other hand, it is almost insensitive to the hardening model [48-50], at least as long as texture evolution is not taken into account in the modeling [51,52]. As a consequence, the impact of the anisotropic potential can be decoupled from the impact of the hardening model.

Several sheet metals have been used in [34] for a detailed comparison of advanced strain-rate potentials. The impact of the identification procedure has been particularly addressed. The aim of the current investigation is to pursue this comparison in terms of finite element predictions of cup heights for cylindrical cup drawing. This section starts with an overview of the material properties and cup drawing test geometry. A detailed mesh sensitivity analysis has been performed in order to investigate the accuracy and limitations of the numerical results. Then, cup heights are predicted for five materials using all plastic potentials presented 
in Section 2.2. These results are used to investigate the ability of the recent Srp2004-18p potential to predict various types of anisotropy; experimental validation data is provided and discussed whenever available, together with a comparison of the strain-rate-potential approach and the yield-criterion approach. Eventually, the effect of the parameter identification technique is evaluated in terms of finite element predictions.

\subsection{Materials and test configurations}

In order to perform a detailed comparison and to enhance the generality of the discussion and conclusions, five different materials have been selected for this study. These are both f.c.c. (several aluminum alloys) and b.c.c. (mild ferritic steel DC06, dual phase steel DP600) sheet metals, exhibiting various kinds of anisotropic behaviors. The study required material data (experimental data from mechanical tests and/or texture data), together with cup drawing test data and cup height results. This information has been experimentally generated in previous work or collected from literature $[11,21,53,54]$.

The geometries of the cup drawing tests are given in Table 1 and Figure 1, together with the hardening parameters, friction coefficient, and an outline of the material data available for the anisotropy parameter identification and validation.

Table 1. Material data and cup drawing test description for the five materials used in the present work.

\begin{tabular}{|c|c|c|c|c|c|}
\hline \multirow{2}{*}{ Materials: } & \multicolumn{3}{|c|}{ Aluminum alloys } & \multicolumn{2}{|c|}{ Steels } \\
\hline & AA6022 & AA2008 & AA2090 & DP600 & DC06 \\
\hline \multicolumn{6}{|c|}{ Cup drawing test geometry; dimensions in [mm] } \\
\hline Sheet thickness & 1 & 1.24 & 1.6 & 1 & 0.8 \\
\hline Sample diameter & 100 & 162 & 158.76 & 100 & 100 \\
\hline Die radius $\left(r_{d i e}\right)$ & 10 & 6.35 & 12.7 & 10 & 10 \\
\hline Punch radius $\left(r_{\text {punch }}\right)$ & 6.8 & 4.78 & 12.7 & 6.8 & 5 \\
\hline Die diameter $\left(D_{\text {die }}\right)$ & 52.5 & 100.08 & 101.48 & 52.5 & 52.5 \\
\hline Punch diameter $\left(D_{\text {punch }}\right)$ & 49.6 & 99.16 & 97.46 & 49.6 & 50 \\
\hline Blank-holder force $[\mathrm{N}]$ & 5000 & 5550 & 5500 & 11000 & 12000 \\
\hline \multicolumn{6}{|c|}{ Material hardening and friction parameters } \\
\hline Yield stress (rolling dir.) [MPa] & 124.2 & 185 & 279.6 & 285.0 & 122.24 \\
\hline$C_{r}$ & 9.5 & 6.14 & 1.7 & 5.6 & 7.8 \\
\hline$R_{\text {sat }}[\mathrm{MPa}]$ & 167.0 & 223 & 488 & 359.6 & 213.6 \\
\hline$C_{x}$ & 146.5 & 0 & 0 & 51.0 & 153.4 \\
\hline$X_{\text {sat }}[\mathrm{MPa}]$ & 34.9 & 0 & 0 & 268.7 & 45.1 \\
\hline Coulomb friction coefficient & 0.1 & 0.123 & 0.1 & 0.1 & 0.2 \\
\hline \multicolumn{6}{|c|}{ Available data for anisotropy identification and validation } \\
\hline Texture & $\mathrm{X}$ & & & $\mathrm{X}$ & $X$ \\
\hline Mechanical tests $(r, \sigma \ldots)$ & $X$ & $X$ & $X$ & $X$ & \\
\hline Cup height profile & & X & $X$ & & \\
\hline
\end{tabular}




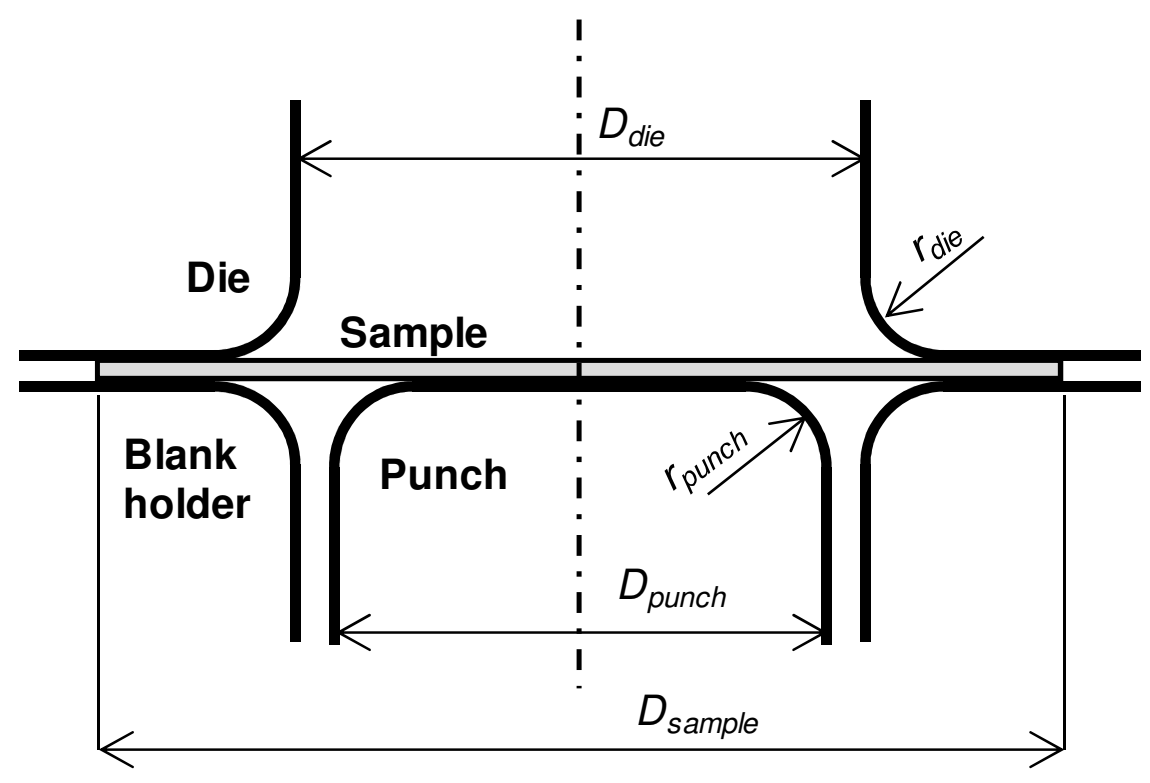

Figure 1. Cup drawing test geometry.

\subsection{Mesh sensitivity analysis}

Finite element simulation of cup drawing is known to depend on the description of the plastic anisotropy in the modeling and on the corresponding parameters. However, when quantitative results are considered, several numerical features and parameters may also have a considerable influence $[44,49,55]$. For the choice of the finite element and mesh density, a sensitivity analysis has been performed that compares two linear solid elements, three inplane mesh densities, and different numbers of element layers through the thickness.

The computer implementation of the material model [33,44] is restricted to solid elements (all the plastic potentials being fully three-dimensional). Moreover, linear elements are recommended whenever nonlinear problems are solved which involve contact evolution. In this category, reduced integration elements are often preferred in industrial applications due to their time efficiency and good overall accuracy. In this investigation, the reduced integration element C3D8R available in Abaqus/Standard is compared to the incompatible-modes enriched, hybrid displacement-pressure element C3D8IH. Three degrees of mesh refinement are compared (see Figure 2) that are designated in the following as "coarse", "fine" and "very fine", respectively ${ }^{1}$. As for the thickness direction, up to three layers of elements have been tested, thus generating meshes containing between 600 to 5500 elements for $1 / 4$ of the sheet. The material model used throughout the mesh sensitivity analysis is Hill's quadratic plastic potential corresponding to the DC06 mild steel (with anisotropy coefficients $r_{0}=2.53, r_{45}=$ $\left.1.84, r_{90}=2.72\right)$.

\footnotetext{
${ }^{1}$ Obviously, these designations have no absolute meaning since a mesh can be considered either coarse or fine depending on the aim of the simulation.
} 
a)

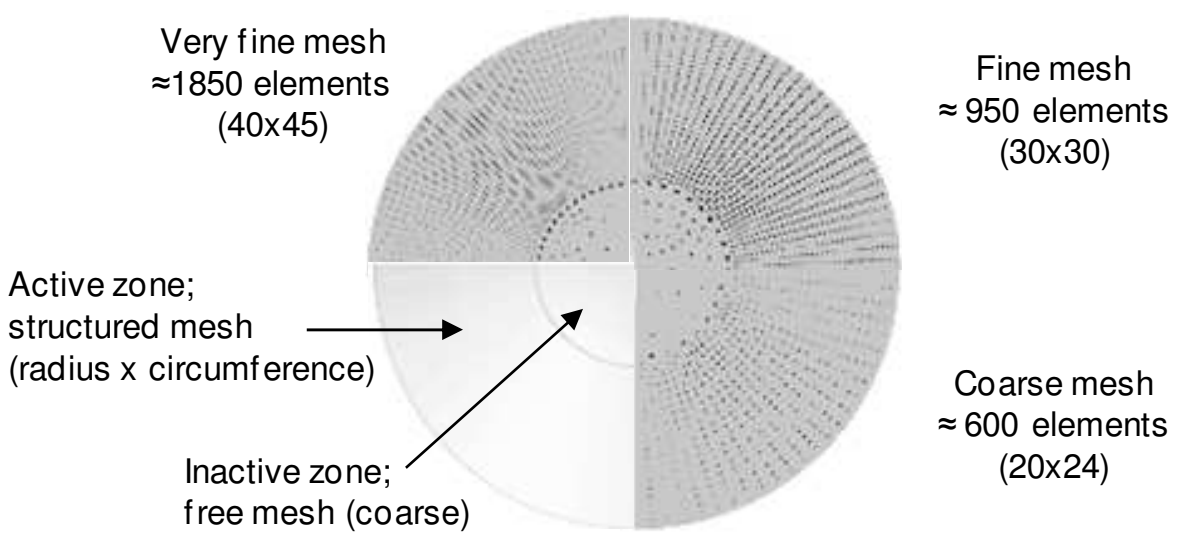

b)

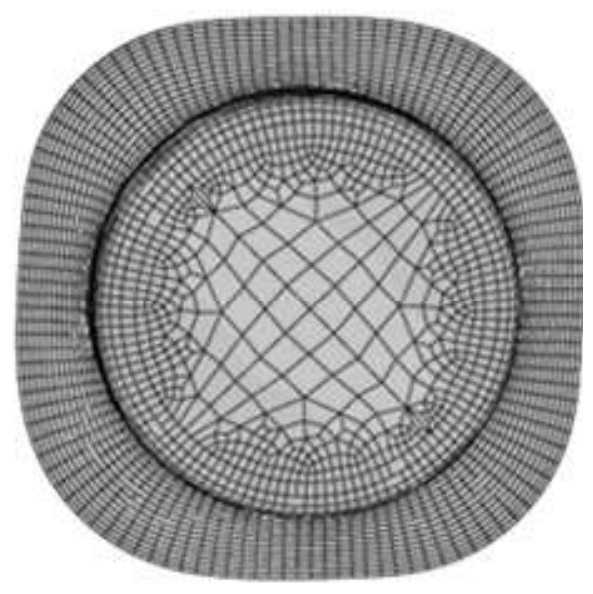

c)

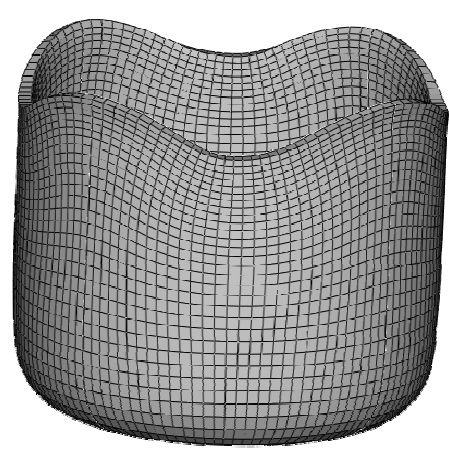

Figure 2. Finite element simulation of cup drawing: a) meshes used for the mesh sensitivity analysis; b) intermediate and c) final configurations predicted with the fine mesh.

Figure 3 shows the mesh sensitivity of the cup height predictions with different elements and mesh densities. It is obvious from Figure $3 \mathrm{a}$ that the predictions with the reduced integration element are very sensitive to mesh density. Finer meshes bring the predictions closer to the converged solution. Moreover, buckling appeared during the simulations with one layer of elements through the thickness (thus not represented on the plot). In contrast, the hybrid element exhibits almost no mesh sensitivity, even when the coarse mesh is used with only one layer of elements. In particular, the number of layers appears to have no influence at all on the cup height, which will be the main simulation result exploited in the following.

The mesh sensitivity of the strain distributions is illustrated by Figure 4. The logarithmic strain in the radial direction is represented as a function of the curvilinear coordinate measured from the center of the cup to its rim, along its outer surface (facing the die), in the rolling direction. The strains are very small on the bottom of the cup and they progressively increase along the cup wall, with a local increase of the radial strain in the punch radius zone due to bending. Since bending induces a strain gradient through the thickness, the values 
represented in Figure 4 correspond to the outer surface of the sheet. Indeed, this is the surface where experimental strains are usually measured in sheet metal forming.
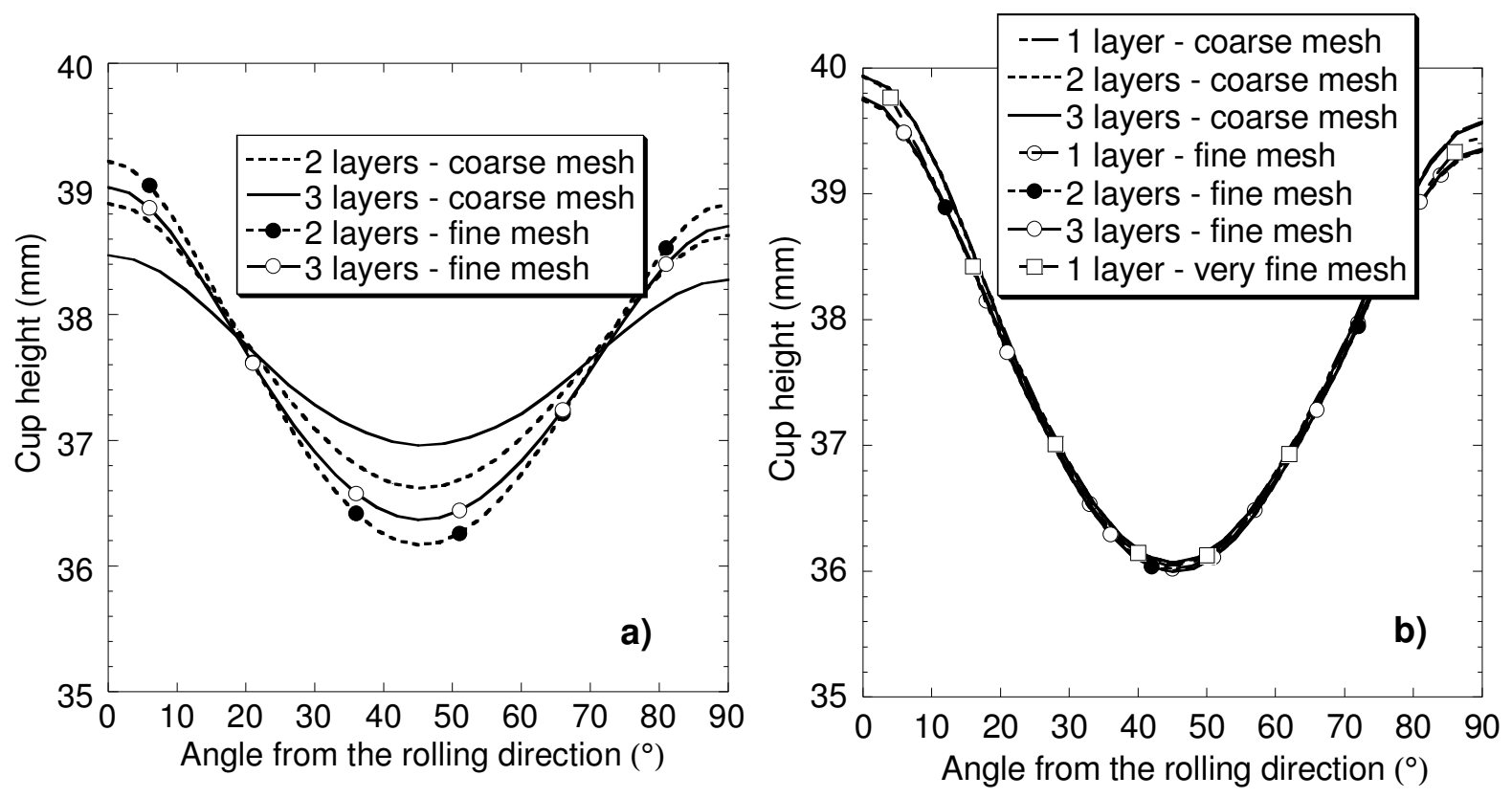

Figure 3. Cup height predictions with the different meshes using a) the reduced integration element C3D8R, and b) the hybrid element with incompatible modes C3D8IH.
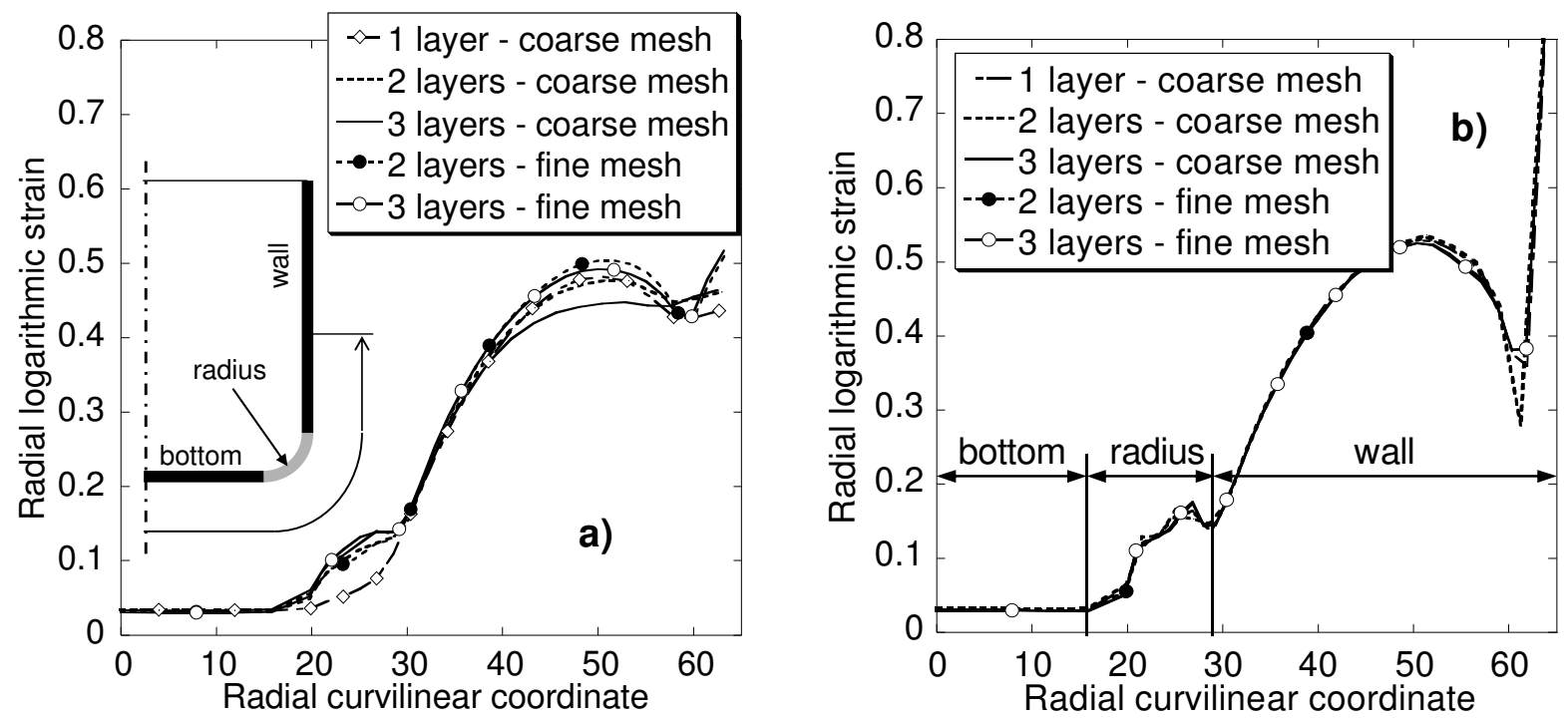

Figure 4. Radial strain distribution along the rolling direction predicted with the different meshes using: a) the reduced integration element C3D8R, and b) the hybrid element with incompatible modes C3D8IH. 
The important mesh sensitivity of the reduced integration element is also observed in terms of strain distribution. The one-layer simulations fail to predict the strain increase due to bending, since only one integration point is present through the thickness. Finer meshes predict strains closer to the converged solution. Again, the hybrid element exhibits almost no mesh sensitivity. These conclusions corroborate well with the cup height predictions, since these two quantities are related.

It is noteworthy that the radial strain presents a considerable jump close to the rim of the cup. Indeed, a strain variation as large as $0.3 \ldots 0.5$ takes place along the last element of the mesh in the radial direction. The explanation for this phenomenon is found at the end of the blank holding phase, when the rim of the flange loses contact with the blank-holder. As shown in Figure 5a and $\mathrm{b}$, the last element in contact with the blank-holder is strongly pinched between it and the die, as it conveys the entire holding force. It is noteworthy that contact on a single node - or a line of nodes - is not recommended and its effect strongly depends on the contact surface definition in the FE code. This pinching effect is also visible in the thickness distribution represented in Figure 5c; indeed, the thickness strongly decreases along the last element, in the rolling and transverse directions, while it increases continuously in the $45^{\circ}$ direction. This phenomenon corresponds to the real pinching of the sample in deep drawing processes with constant holding force. Figure $5 \mathrm{~d}$ shows experimental evidence of this phenomenon, which often exaggerates any misalignment between the sample and the tools (here, the right-hand side of the cups has been pinched more strongly). Depending on the strength of the material and the holding force, the occurrence of pinching can modify the shape of the ears, which do not depend only on material anisotropy but also on such process parameters.

Finally, the mesh sensitivity of the predicted stresses is also investigated. Figure 6 shows the equivalent stress distributions on the inner surface of the cup for the different meshes and finite elements. When buckling has occurred, the stress distribution has been strongly deviated and could not be compared to the remaining ones.

While the reduced integration element exhibits relatively small mesh sensitivity in terms of predicted stresses, a much larger sensitivity is observed for the hybrid element. The in-plane refinement of the mesh does not really influence the stresses, yet the number of layers has a very important impact since the maximum stress is reduced by $25 \%$ between the simulations with three layers and only one layer, respectively. One possible explanation for this behavior could be the technique used in the FE code to extrapolate stresses to the surface of the part. Indeed, when one layer of elements is used, the stresses are calculated at two integration points along the thickness and then extrapolated to the two surfaces. A non-negligible stress gradient is observed between the inner and the outer surface of the cup even when one single hybrid element is used.

In summary, the cup height predictions are mesh-insensitive whenever the hybrid element is used, and one single layer of elements is sufficient in any case. The "fine" mesh will be used for all the following simulations, with one layer of elements through the thickness. While the (radial) strain distributions are fairly insensitive to the mesh, the predicted stresses are much more sensitive and should not be used for the comparison of different models. It has also been observed that process parameters such as pinching, holding force, clearance between the punch and the die - as well as numerical parameters like the value of friction coefficient or contact algorithm parameters - have a non-negligible influence on the cup heights, which 
cannot be considered as a pure effect of material anisotropy (although this is their main origin).

a)

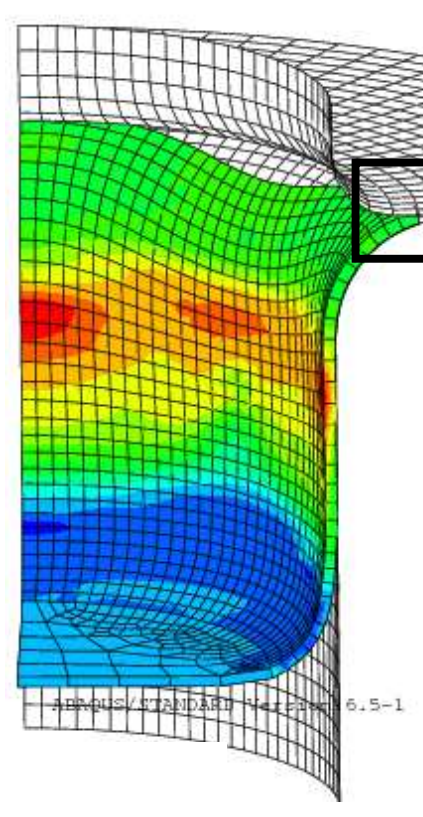

d)
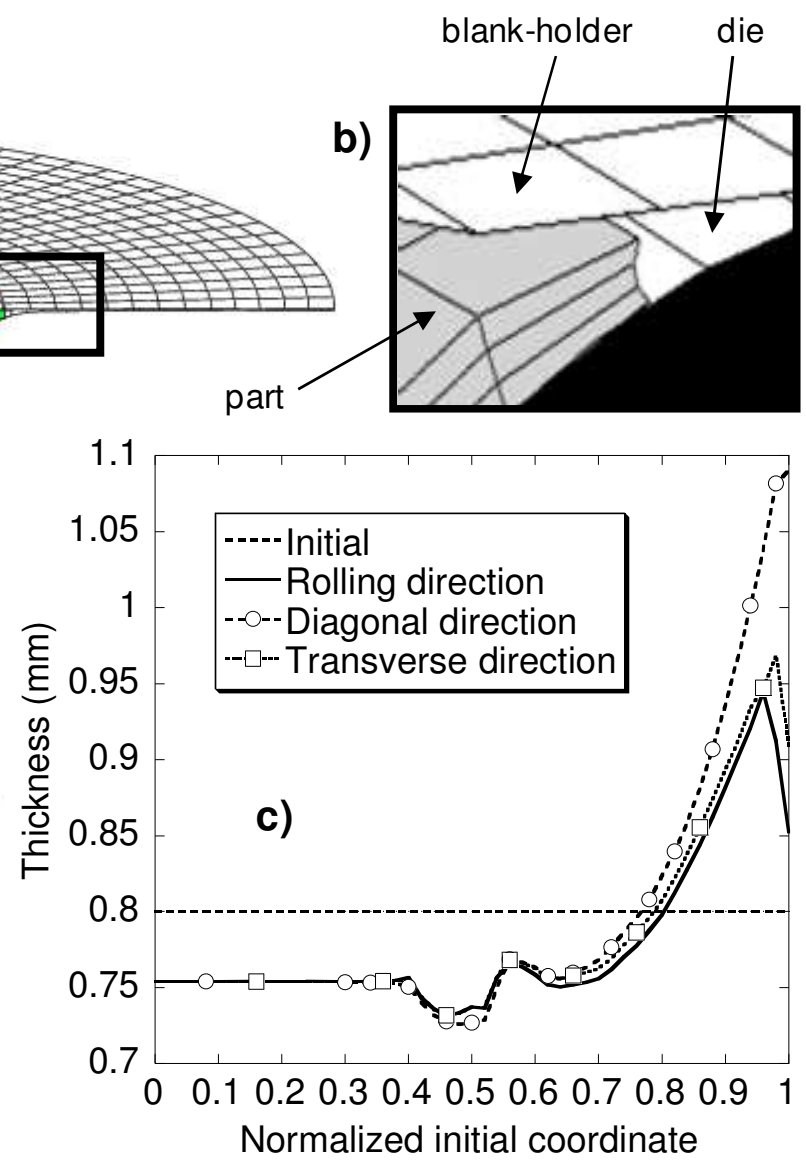

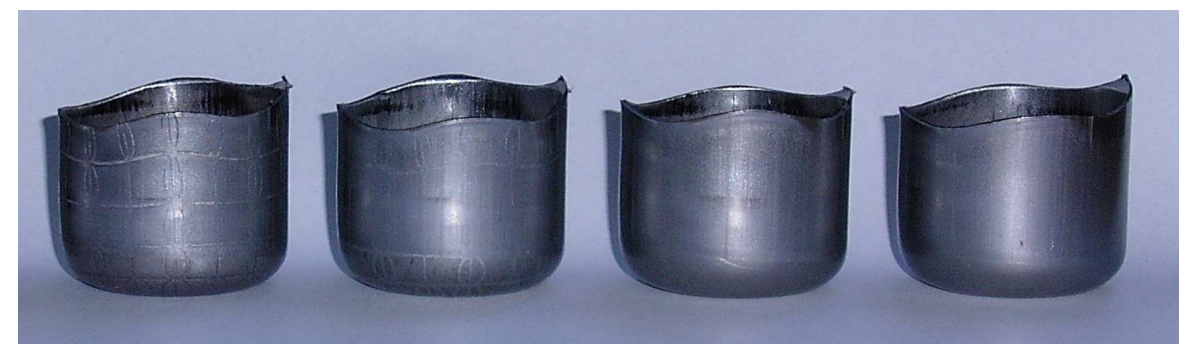

Figure 5. Pinching effect at the end of the blank holding step: a) as predicted by Abaqus; b) detail of the one-element pinched zone; c) radial thickness distribution along the rolling direction, the transverse direction, and at $45^{\circ}$ with respect to the rolling direction; d) experimental cups in mild steel which exhibit pinched ears (top right). 

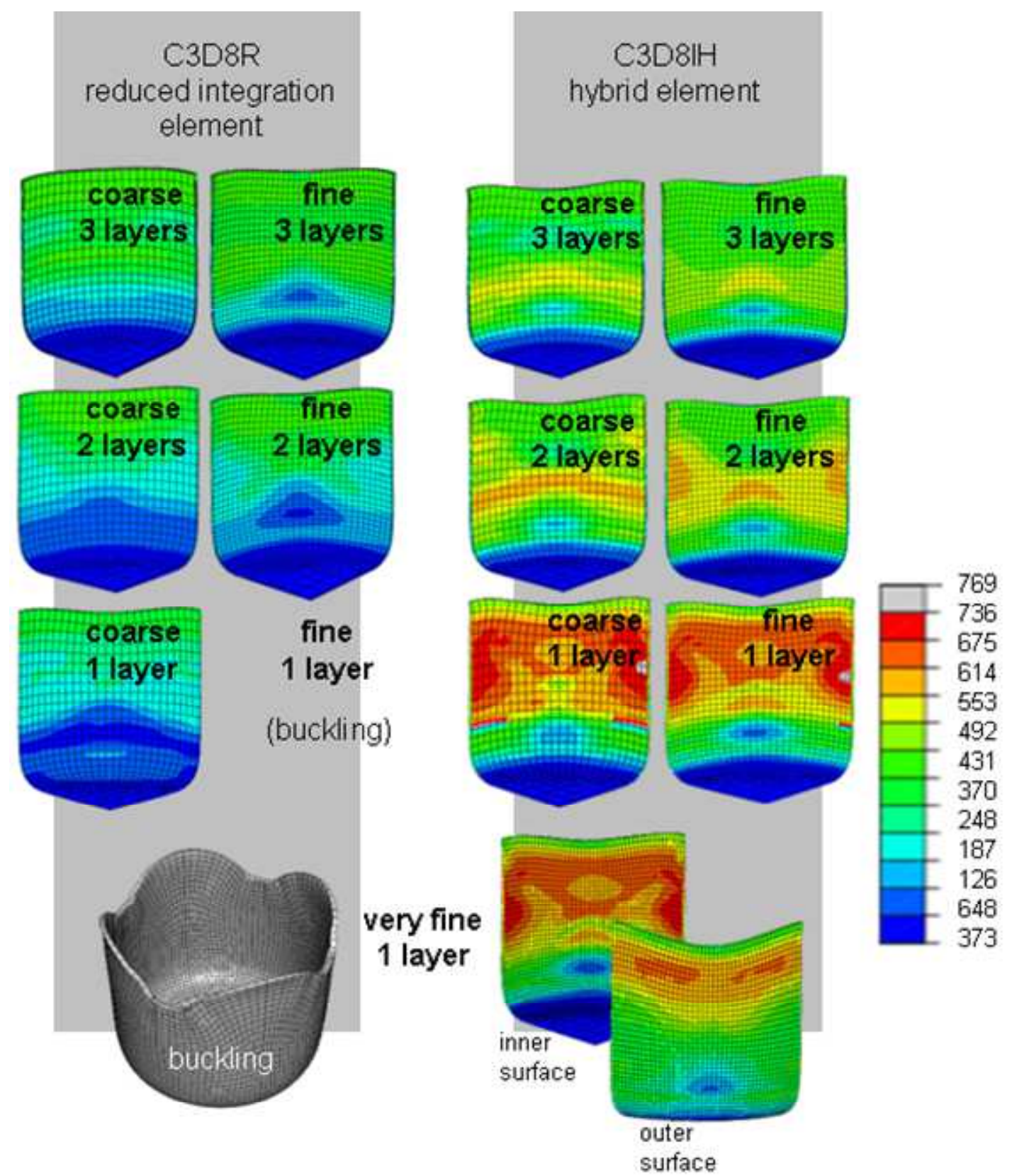

Figure 6. Mesh sensitivity of the equivalent stress distribution for the cup drawing simulations with reduced integration elements (left) and hybrid displacement-pressure elements (right), for different mesh densities and one, two, or three layers of elements through the thickness. 


\subsection{Results and analysis of the cup drawing simulations}

The correctness of the numerical implementation of the constitutive model and its state update algorithm have been validated [33] in the case of Hill's potential. The aim here is to investigate the accuracy of the finite element predictions for the other strain-rate potentials, especially Srp2004-18p. The results are compared to experiments and to the predictions if the Yld2004-18p stress potential. The computational efficiency of the proposed models is also addressed, together with their accuracy and their ability to describe the plastic anisotropy of various materials.

\subsubsection{Comparison to experiments and to the Yld2004 criterion predictions}

Chung and Shah [9] provided the experimental cup profile and the $r$-value in-plane variation for the cup drawing of the AA2008 material. Figure 7 shows these results together with the predictions obtained in the current investigation with three potentials. The in-plane variation of the anisotropy coefficient is very well predicted by the three potentials, the three predictions being very close to each other. However, the cup profiles predicted by Quartus and Srp2004-18p are different from those of Hill's potential, in spite of their close predictions of $r$-values. This is not surprising in view of the more detailed comparison given in [34]. There, it has been observed that when mechanical tests are used for parameter identification, the Quartus and Srp2004-18p yield surfaces are very close to each other (Figure 18 in [34]) and the corresponding error functions are almost identical (Figure 11 in [34]), while the one corresponding to Hill is much larger, indicating that its predictions of the stress distribution and possibly the biaxial stress point are less accurate. Hill's predictions of cup height overestimate the amplitude of the ears, while they better locate the valley between them.

Figure 7 seems to confirm the (loose) similarity between the $r$-values $r(\alpha)$ and the cup heights $h(\pi / 2-\alpha)$, reported in several papers. Based on this assumption, Yoon et al. [11] have developed an analytical formula for the cup height distribution, whose predictions are also given in Figure 7. With this analytical formula, the ear locations are well predicted but the cup height is not accurate.

A second material is used for the experimental confrontation; this is the AA2090 ,aluminum alloy which exhibits six ears [11]. The corresponding cup height predictions are given in Figure 8, along with the $r$-values. As expected, Hill's potential fails in predicting more than four ears. However, the height predictions of the quadratic potential are surprisingly accurate (except for the two "missing ears", which happen to be relatively small), in spite of a poor prediction of the $r$-values. Srp2004-18p predicts six ears at the good locations, however the years at $0^{\circ}$ and $180^{\circ}$ are underestimated and hardly distinguishable; its predictions lie close to the ones obtained by Yoon et al. [11] with Yld2004-18p. Quartus predicts eight ears, although two of them are almost negligible (at $90^{\circ}$ and $270^{\circ}$ ) and its overall predictions are very similar to the ones of Srp2004-18p and Yld2004-18p. Finally, the analytical formula of Yoon et al. [11] predicts the number and the location of the ears but their amplitude is underestimated. 

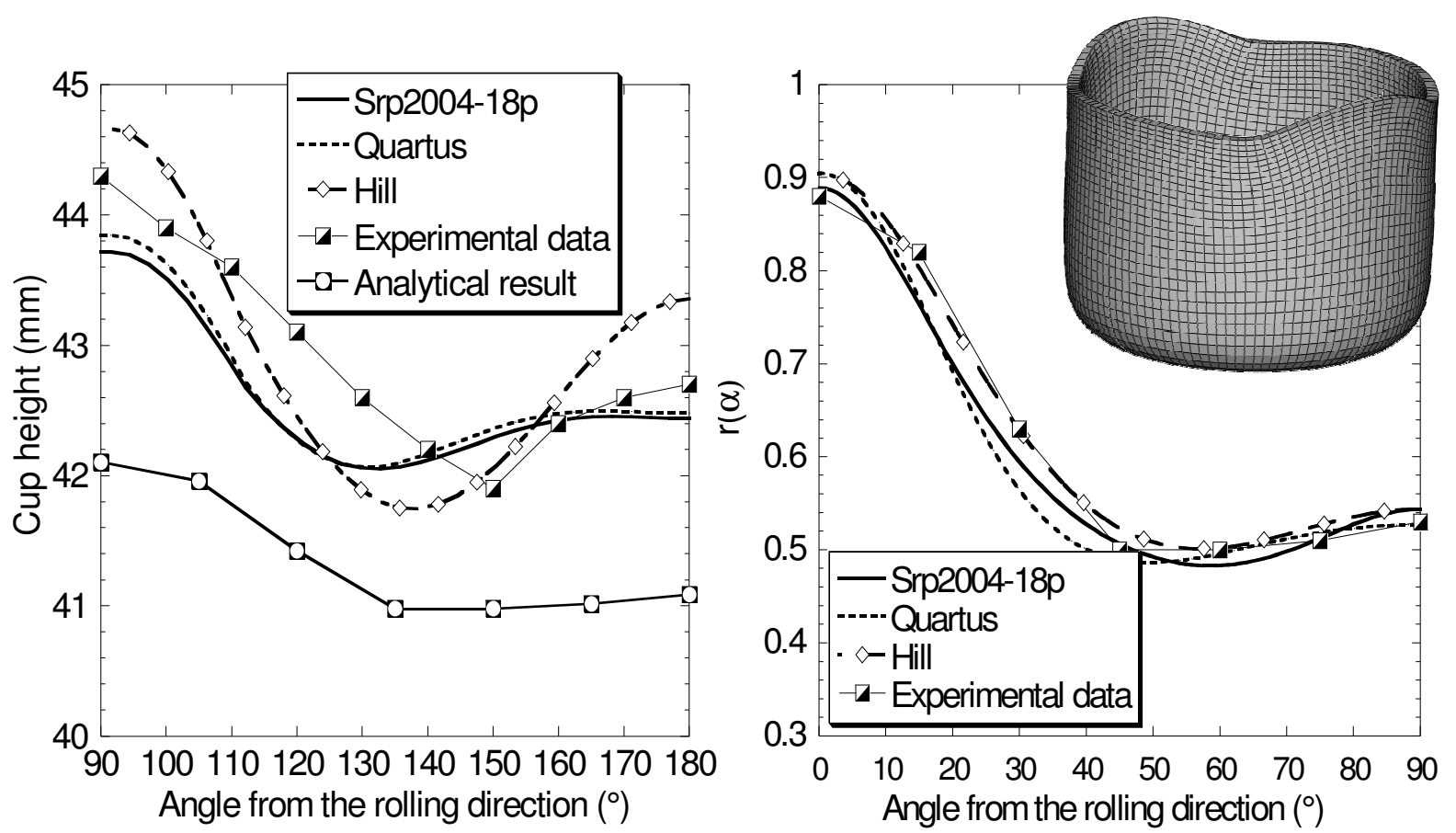

Figure 7. Cup height and r-value predictions for the AA2008 aluminum alloy.

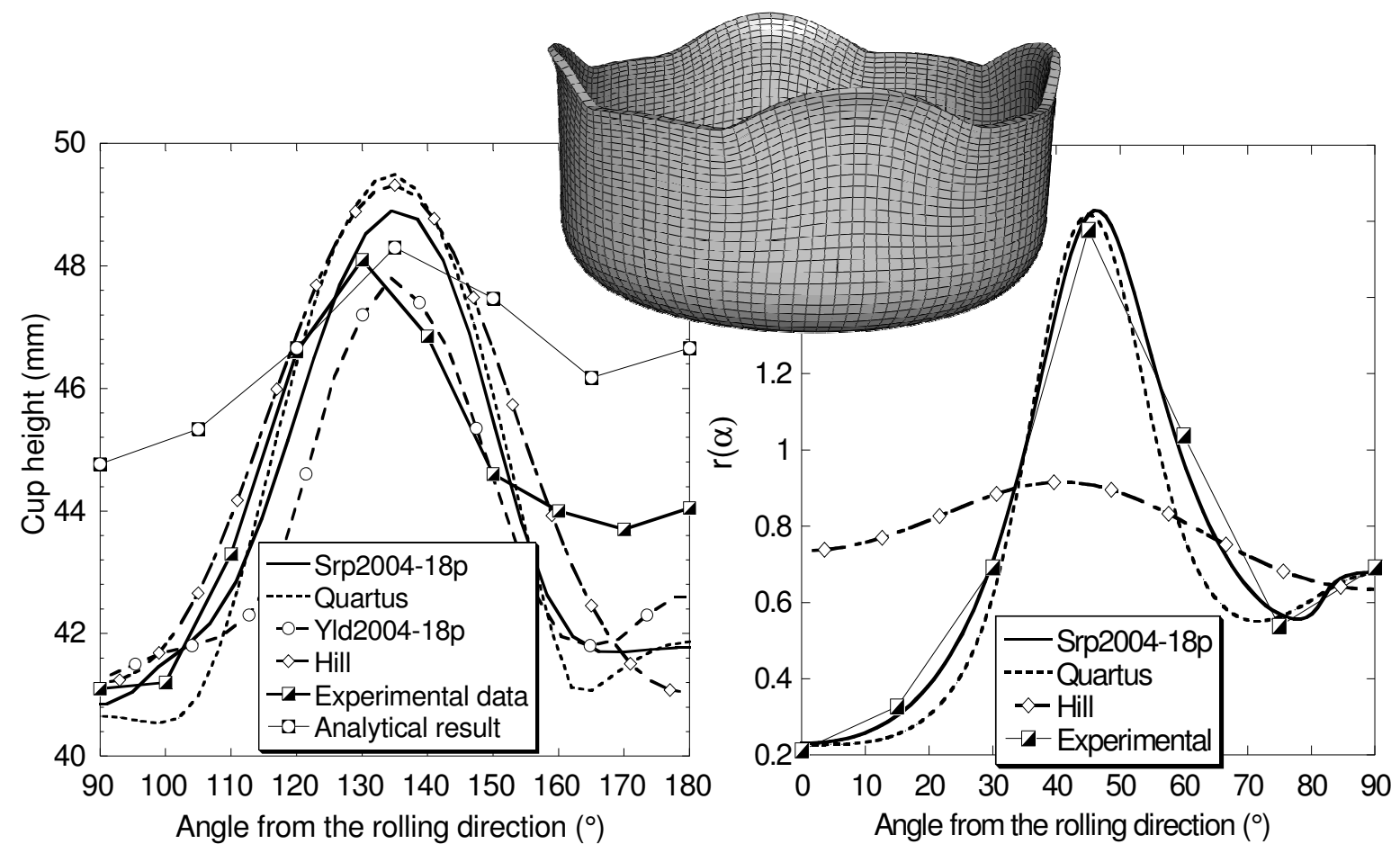

Figure 8. Cup height and r-value predictions for the AA2090 aluminum alloy. 


\subsubsection{Ability of Srp2004 to describe various types of anisotropy}

The Srp2004-18p model is further investigated in this section since it has been seldom applied in the literature to address its predictive capabilities in FE simulations. Figure 9 reviews the cup drawing results obtained for some of the materials investigated in this study. As commonly accepted, the shape of the ears appears to follow loosely the shape of the $r(\alpha)$ graph and several four-ear cups are correctly simulated, as well as the six-ear cup already discussed.

Two more simulations are used to further illustrate the flexibility of the Srp2004-18p potential. A virtual material has been generated by enforcing the $r$-value variation depicted in Figure 10a. After parameter identification based on this data, the cup drawing simulation allowed for the prediction of eight ears. Finally, the tensile stress variation of an AA5182 aluminum alloy [34] depicted in Figure 10b has been used to identify the parameters of Srp2004-18p. As shown in Figure 10b, the simulation leads to a ten-ear cup profile. Therefore, up to ten ear cup profiles can be predicted with Srp2004-18p.

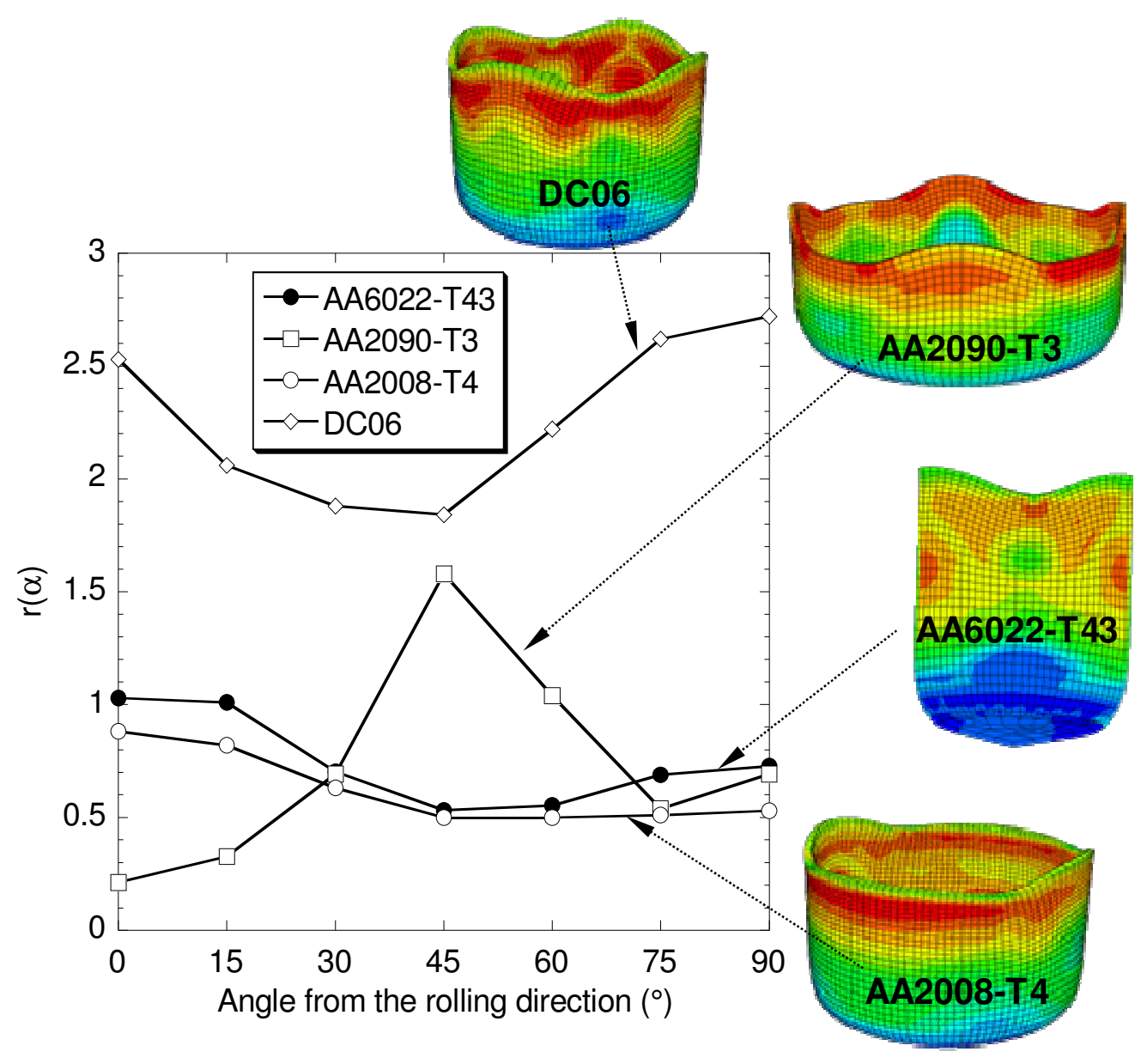

Figure 9. Experimental r-values and predicted cup heights for the different materials, using Srp2004-18p with material parameters identified by mechanical tests. 
a)
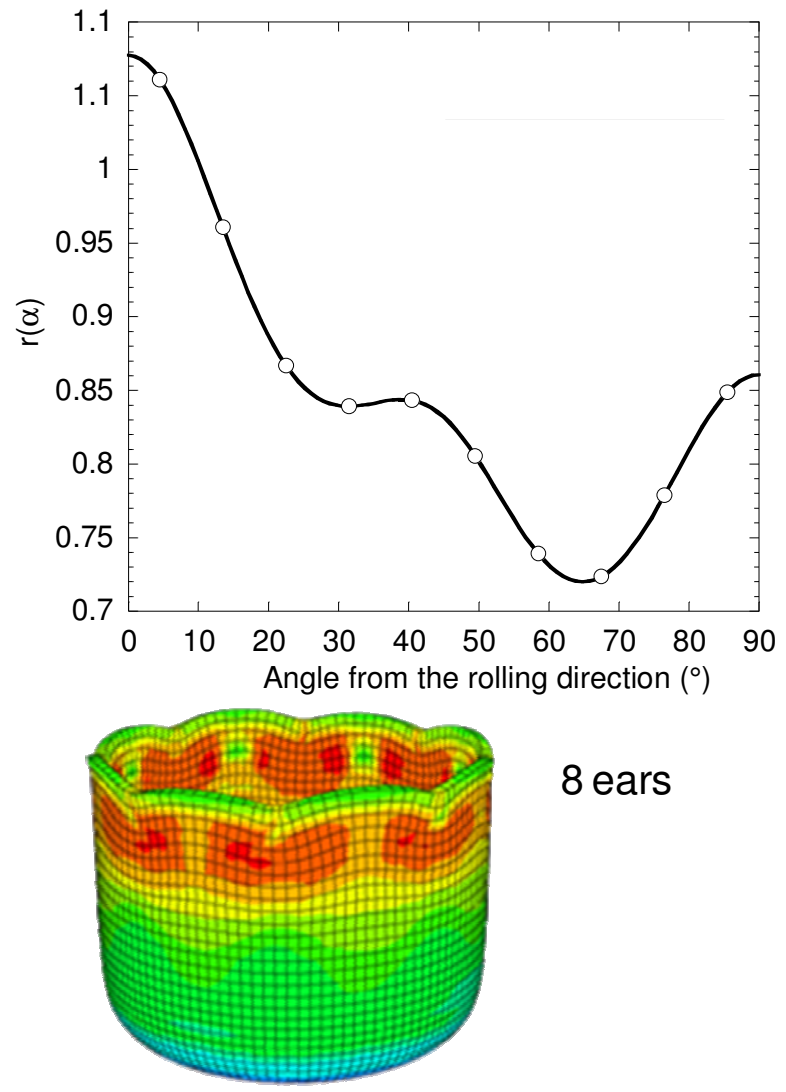

b)
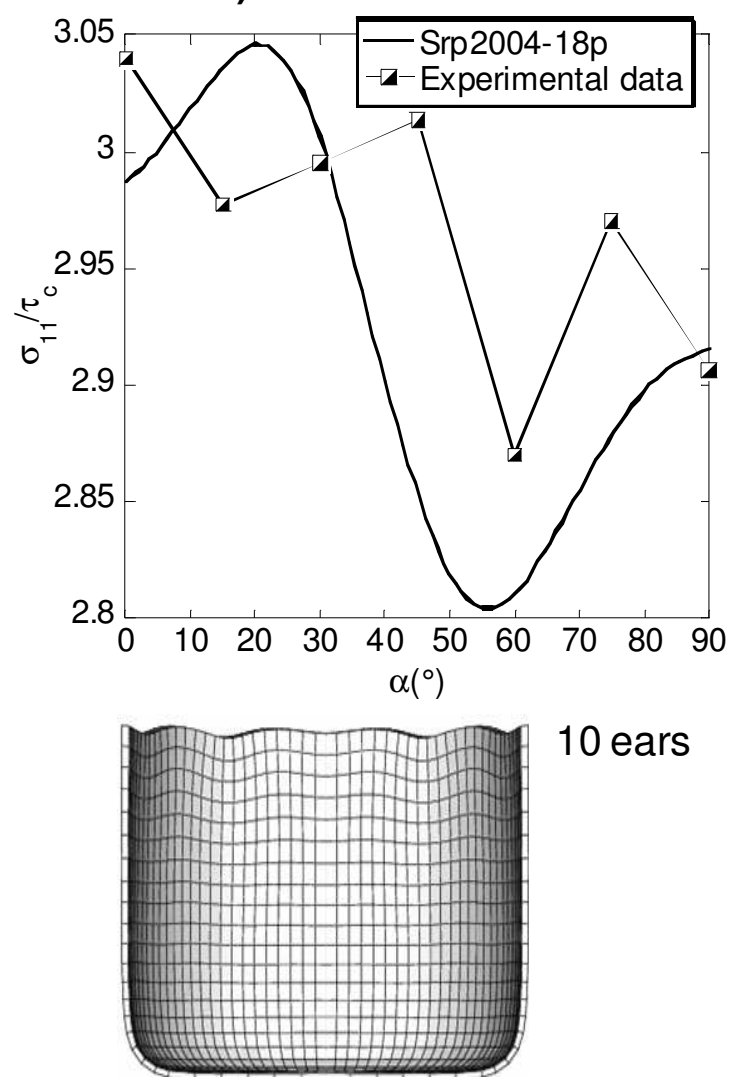

10 ears

Figure 10. Cup drawing simulations with Srp2004-18p, for a) a virtual material, exhibiting eight years and b) the AA5182 aluminum alloy, exhibiting ten ears.

\subsubsection{Comparison of CPU time}

The previous comparisons have shown the superior ability of Srp2004-18p and Quartus to predict six, eight, or more cup ears and to reproduce the $r$-values more accurately. However, the cup height predictions of the quadratic potential are surprisingly good for the analyzed tests and materials. In order to put this comparison into perspective, Table 2 shows the computing time required by the cup drawing simulations for two materials when various potentials are used (the calculations have been run on a PC computer with a Pentium $1.8 \mathrm{GHz}$ dual core processor). The quadratic potential is also compared to the Abaqus built-in Hill' 48 yield criterion, which is taken as reference. The quadratic potentials give not only identical predictions [33], but the computing time is almost the same (less than 5\% larger in the strainrate potential case). However, the use of the Quartus potentials increases the computing time by a factor of two. The cost of the Srp2004-18p potential is even higher, going from a factor of 9 up to 24, depending on the nonlinearity of the potential. Indeed, the shape of the yield surface for the AA6022 material requires a systematic activation of the sub-stepping procedure, thus increasing the computing time considerably. This is definitely a part of the computer code that might be improved in the future. 
Table 2. Computing time impact of the different strain-rate potentials.

\begin{tabular}{cccc}
\hline Potential & \multicolumn{2}{c}{ CPU time } & $\begin{array}{c}\text { Number of } \\
\text { increments }\end{array}$ \\
\hline Hill48 (Abaqus) & DP600 & AA6022-T43 & 240 \\
Hill48 (Umat) & 3h37' (100\%) & 3h31' (100\%) & 240 \\
Quartus & 8h42' $(252 \%)$ & 3h39' (104\%) & 238 \\
Srp2004-18p & 31h49' $(922 \%)$ & 84h5' (166\%) & 231 \\
\hline
\end{tabular}

Figure 11 recalls the gains in accuracy obtained with each of these potentials, as calculated in [34] during the parameter identification procedure. This theoretical accuracy gain is balanced by its cost (Table 2) as well as its practical relevance e.g., in cup drawing simulations, as reported in the previous sections.

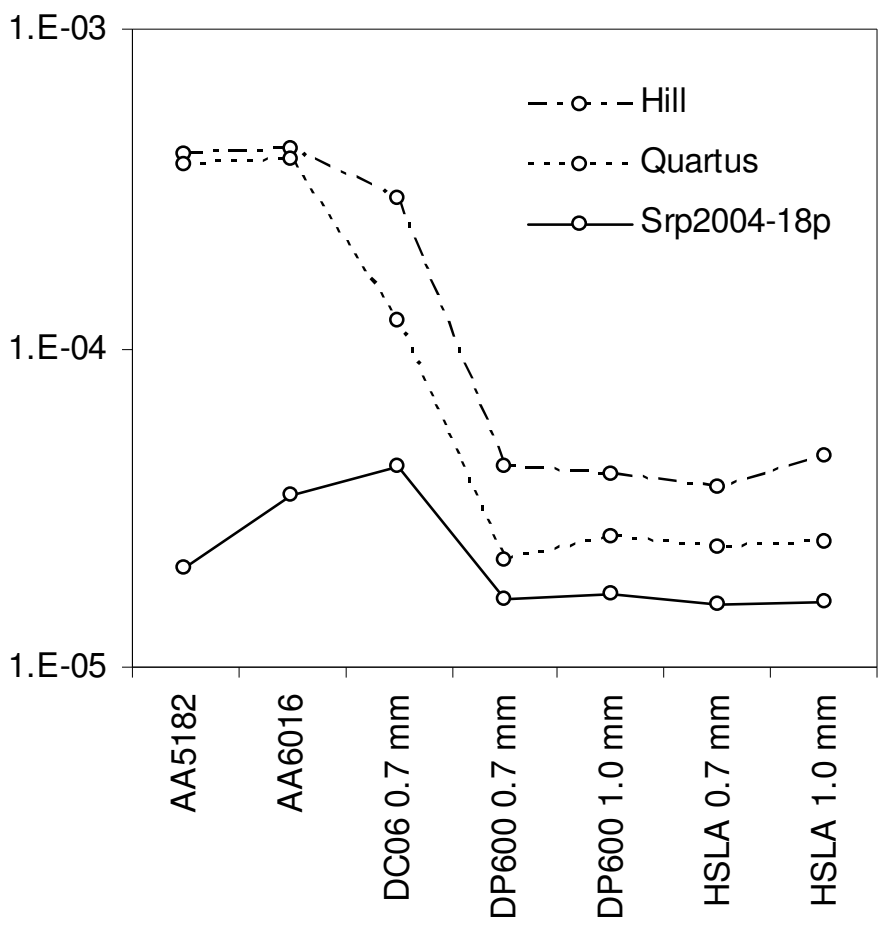

Figure 11. Texture-based error functions, Eq. (19), for the three strain rate potentials and for several sheet materials investigated in [32]. 


\subsection{Impact of parameter identification on FE simulation results}

The previous simulations have shown that the choice of the strain rate potential may have an important impact on the predicted cup profile. It has been also recognized for a long time that the data used for the parameter identification may play an important role and may be more important than the model itself $[51,52,54,56]$. In the case of Hill's yield criterion, for example, the parameter identifications based on the $r$-values or based on the uniaxial yield stresses, respectively, are known to lead to different results. However, for more flexible potentials, the number of parameters becomes so large that all the experimental data available is used for the identification. Moreover, there is no analytical link between measured quantities and model parameters (as is the case for the quadratic model); the parameters are usually automatically identified by an inverse method.

In [34], the identification method based on mechanical tests has been compared to the identification method based on texture data and a micromechanical model. The latter has proven to be valuable tool for classifying in a rigorous way different potentials, in terms of their mathematical ability to fit different types of anisotropy. It also provides the only means available to identify material parameters related to out-of-plane components of the plastic strain rate tensor. It has been noticed however that, as their accuracy solely depends on the predictive capacity of the micromechanical model, in some situations the results may poorly fit mechanical test results. Similar conclusions have been published recently by Grytten et al. [57].

Therefore, the impact of the identification technique on the FE results is addressed in the following. Figure 12 compares the $r$-values of the dual-phase steel predicted by Srp2004-18p, for each of the two identification techniques. The $r$-values predicted by the TBH model are different from the experimental ones, although both show maxima in the rolling and transverse directions. It is clear from these plots that when the identification is performed with the TBH model, the phenomenological predictions lay closer to the $r$-values predicted by the TBH model, even though in this case the $r$-values are not used as data for identification (see [34]) for more details on the texture-based identification procedure). When mechanical tests are used, the predictions fit closer to the experiments. In this particular case, one can note that the optimal fit obtained by the automatic identification caused slight deviation of one maximum of the curve, which lies at $72^{\circ}$ instead of $90^{\circ}$ with respect to the experimental data. In terms of curve fitting, this is the optimal solution that could be achieved with the given mathematical model. 

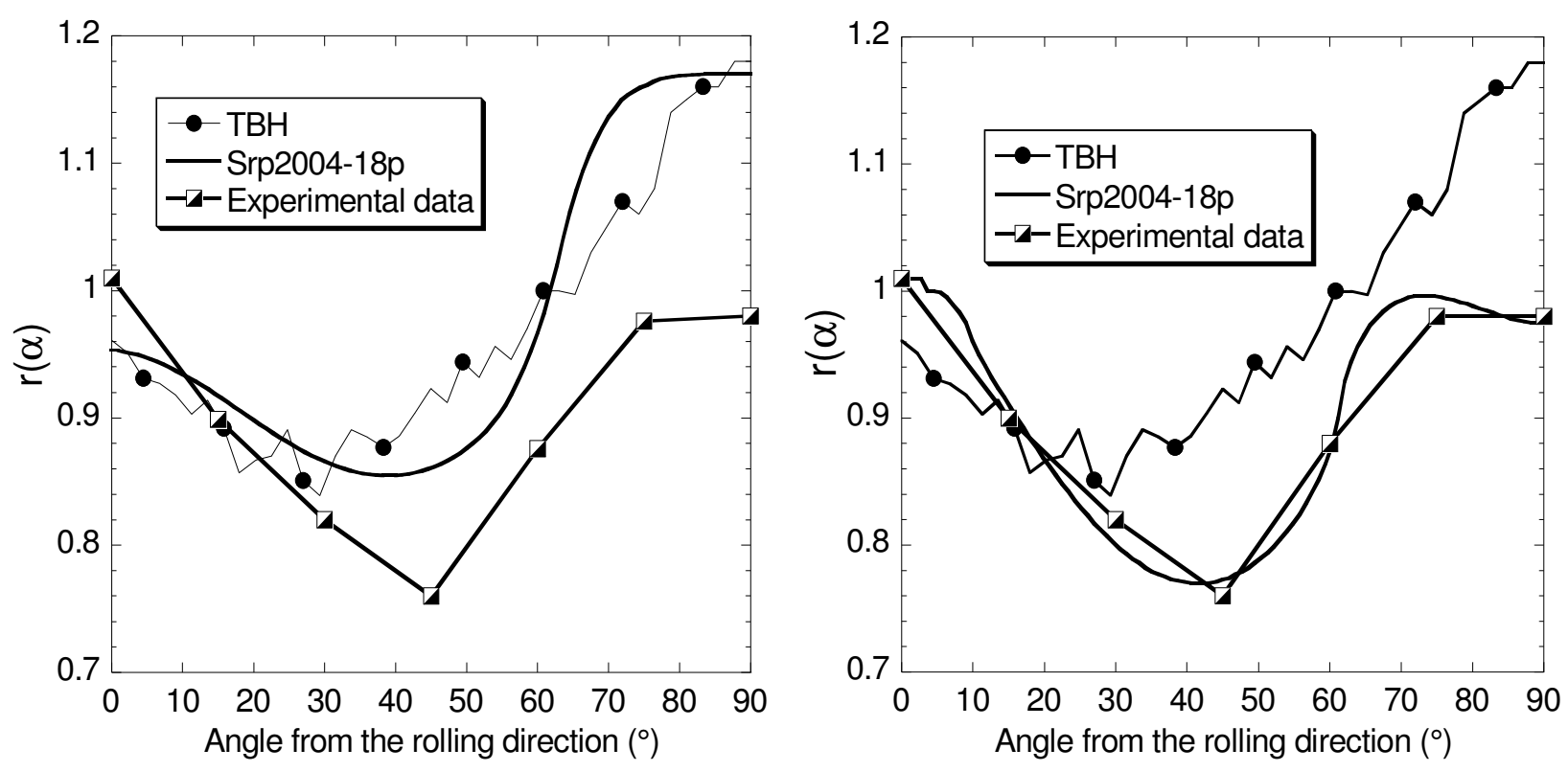

Figure 12. Impact of the identification technique on the predicted r-values for the Srp2004$18 \mathrm{p}$ potential and comparison to experimental and micromechanical results; a) identification using texture data, and b) identification using experimental data from mechanical tests.

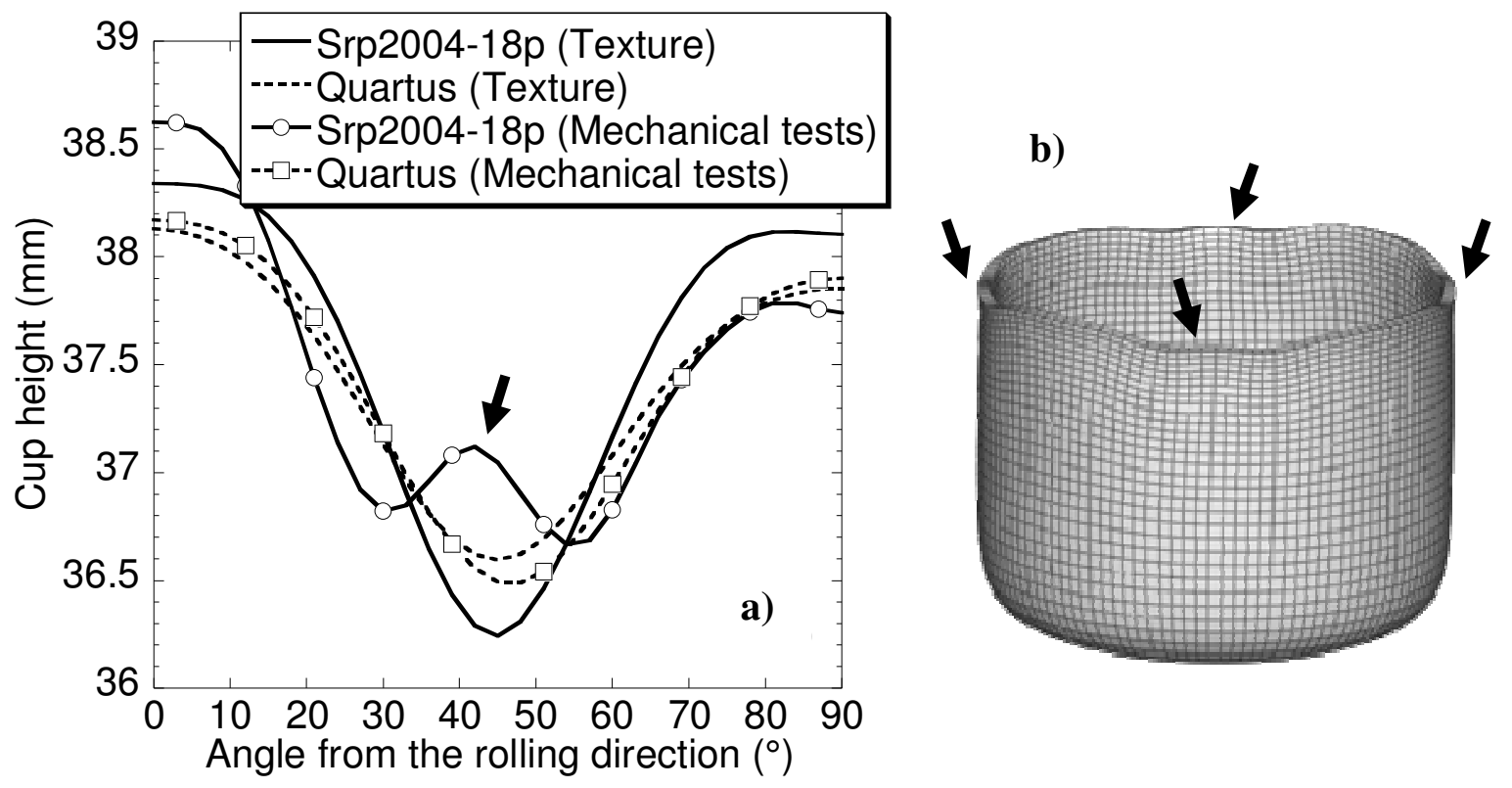

Figure 13. Numerical simulations for the DP600 steel sheet: a) cup height predicted by Srp2004-18p and Quartus with the two identification techniques; b) eight-ears profile predicted with Srp2004-18p and mechanical tests identification (arrows indicate the four unrealistic ears). 
In order to further investigate this issue, Figure 13 compares the cup profiles for the dual phase steel sheet, as predicted by Quartus and Srp2004-18p when both identification techniques are used. The two predictions with Quartus are very close to each other, predicting four ears as suggested by the in-plane anisotropy graph. The texture-based Srp2004-18p is slightly different, yet has the same characteristics. In turn, the experiment-based Srp2004-18p predicts supplementary, unrealistic ears at approximately $42^{\circ}$ with respect to the rolling direction. The height of these ears is very small since the anisotropy of dual phase steels is not very large; however, it is surprising that the number of ears is not correctly predicted. As one can see in Figure 14 ( $a$ and $b$ ), this is not an exceptional case, and it is not specific to the Srp2004-18p potential only. Indeed, the experiment-based Quartus predictions for the AA6022 aluminum alloy sheet exhibit eight ears, while the texture-based Quartus and the two Srp2004-18p versions correctly predict only four ears. The four-ear profile is doubtlessly suggested by the $r$-value distribution (Figure 14c), which is moreover fairly classical and easy to fit with almost any anisotropic model. However, the yield stress distribution (Figure 14d) associated to it is different in shape and is almost a straight line. The automatic identification procedure generated a best-fit solution for which the uniaxial yield stresses oscillate in the neighborhood of the experimental values, thus minimizing the objective function at most. As a consequence, the predicted cup profile lies relatively close to the correct one but with two spurious oscillations at $0^{\circ}$ and $90^{\circ}$. 


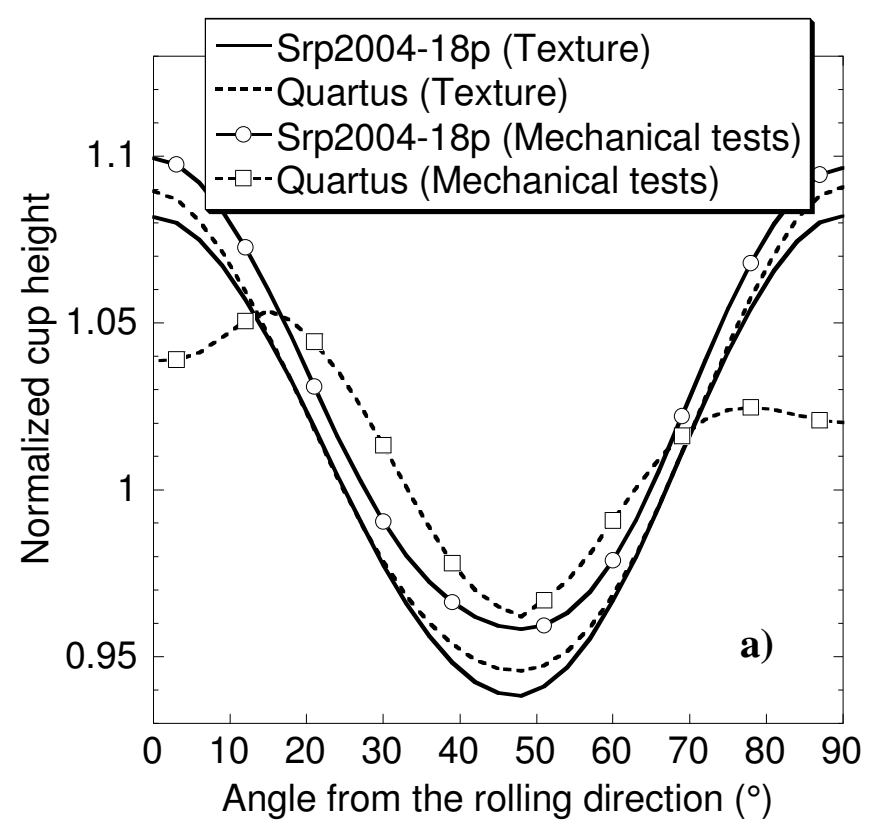

b)
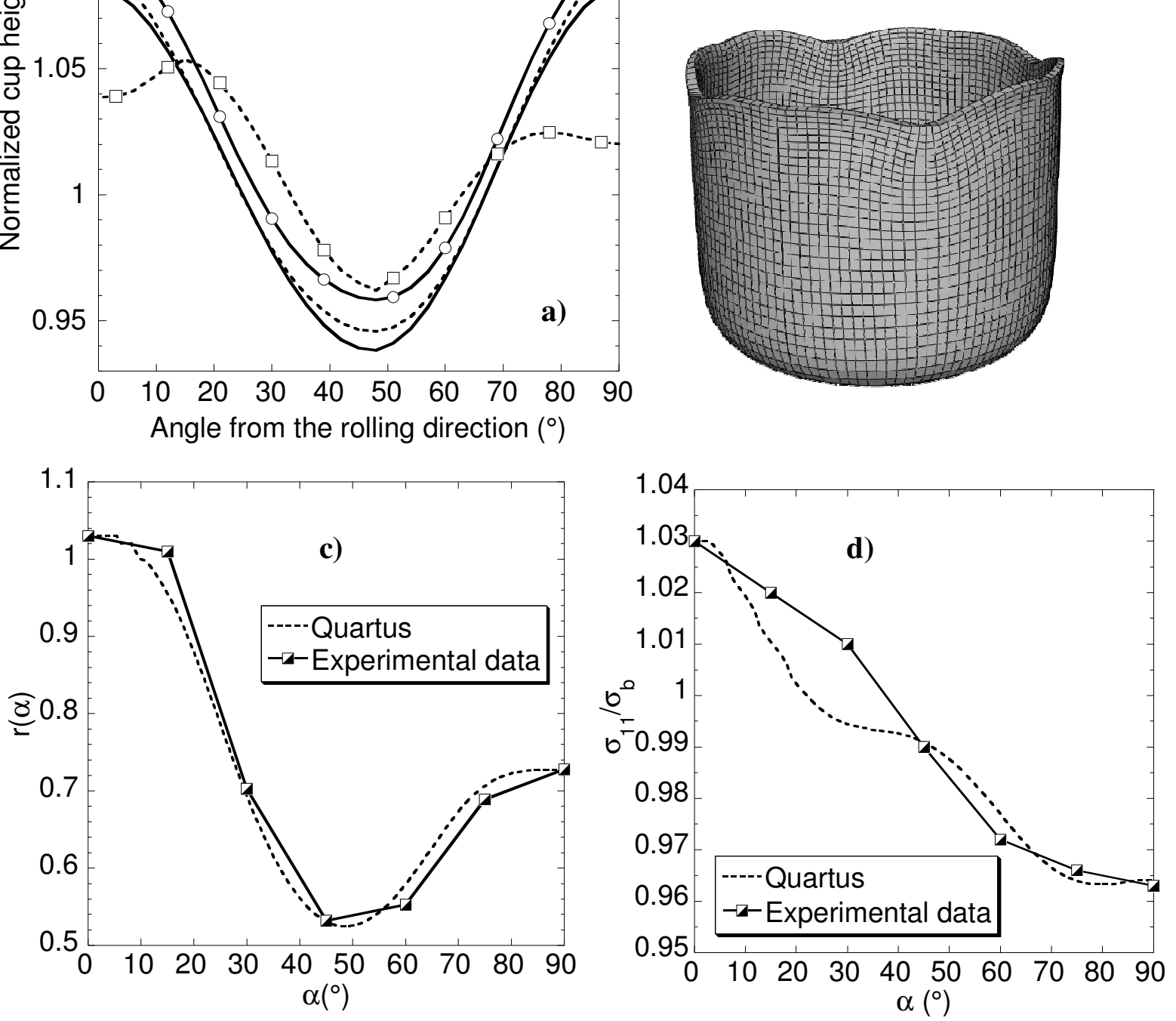

Figure 14. Numerical simulations for the AA6022 aluminum alloy sheet: a) cup height predicted by Srp2004-18p and Quartus with the two identification techniques; b) eight-ears profile predicted with Quartus and the mechanical tests identification; c) r-values and d) uniaxial stresses (normalized by ) as predicted by the experiment-based Quartus model. 
In spite of these two extreme examples, the experiment-based identification technique consistently provides parameter sets whose predictions better reproduce the experimental results (as compared to the TBH-based parameters). Therefore, these examples do not represent a criticism against the experiment-based identification, but rather a warning regarding the automatic parameter identification approaches (which are more and more popular), whatever reference data is used (either texture or experiments). As one can see in Figure 15, the choice of the identification method may have considerably more impact on the predicted results (here, the logarithmic radial strain) than the choice of the potential. This is especially true for the more flexible models (e.g., Quartus, Srp2004-18p), while Hill's model exhibits small sensitivity to the identification method. In this framework, the very large number of data used by the texture-based identification seems to prevent the solution from aberrant oscillations. For the Quartus potential, it has already been shown to lead less often to parameter sets that violate the convexity conditions [34]. However, in the case of the experiments-based identification of advanced potentials, the number of reference data is very often equal to the number of parameters to identify; this may lead to oscillating solutions between the experimental points. Similar conclusions have been drawn by Bouvier et al. [56] for the identification of yield criteria with reduced sets of experimental data. The authors suggest increasing the number of reference data-points with pseudo-experiments generated by the Hill model. While these data are given a smaller weight so that they do not deviate the solution significantly from the real experiments, they prevent aberrant results from being accepted. In our case, the combined experiments-texture identification method proposed in [34,58] provides a consistent and accurate method to overcome such aberrant solutions; in the meantime, it allows for the identification of the potential's parameters corresponding to the out-of-plane terms of the plastic strain rate tensor which cannot be identified by experiments alone. The "smoothing" effect of the texture-based identification is obvious from the resulting yield surfaces [34]. Figure 16 shows the predicted yield surfaces for the AA6022 aluminum alloy when one or the other identification methods are used. The experiments-based models lay close to the experimental uniaxial stress points and to the experimental biaxial stress point (at least for Quartus and Srp2004-18p). They also predict the experimental $r$-values much better than the texture-based versions. On the other hand, however, the corresponding yield surfaces are fairly different from each other since very few data points are available for the identification in the plane $\left(\sigma_{1}-\sigma_{2}\right)$. In contrast, the texture-based identification makes use of numerous, uniformly spread data points and thus generates yield surfaces that are much closer to each other. It is likely that the same conclusions apply to other 2D representations of the yield surface, including shear terms, where almost no experimental point is present. 


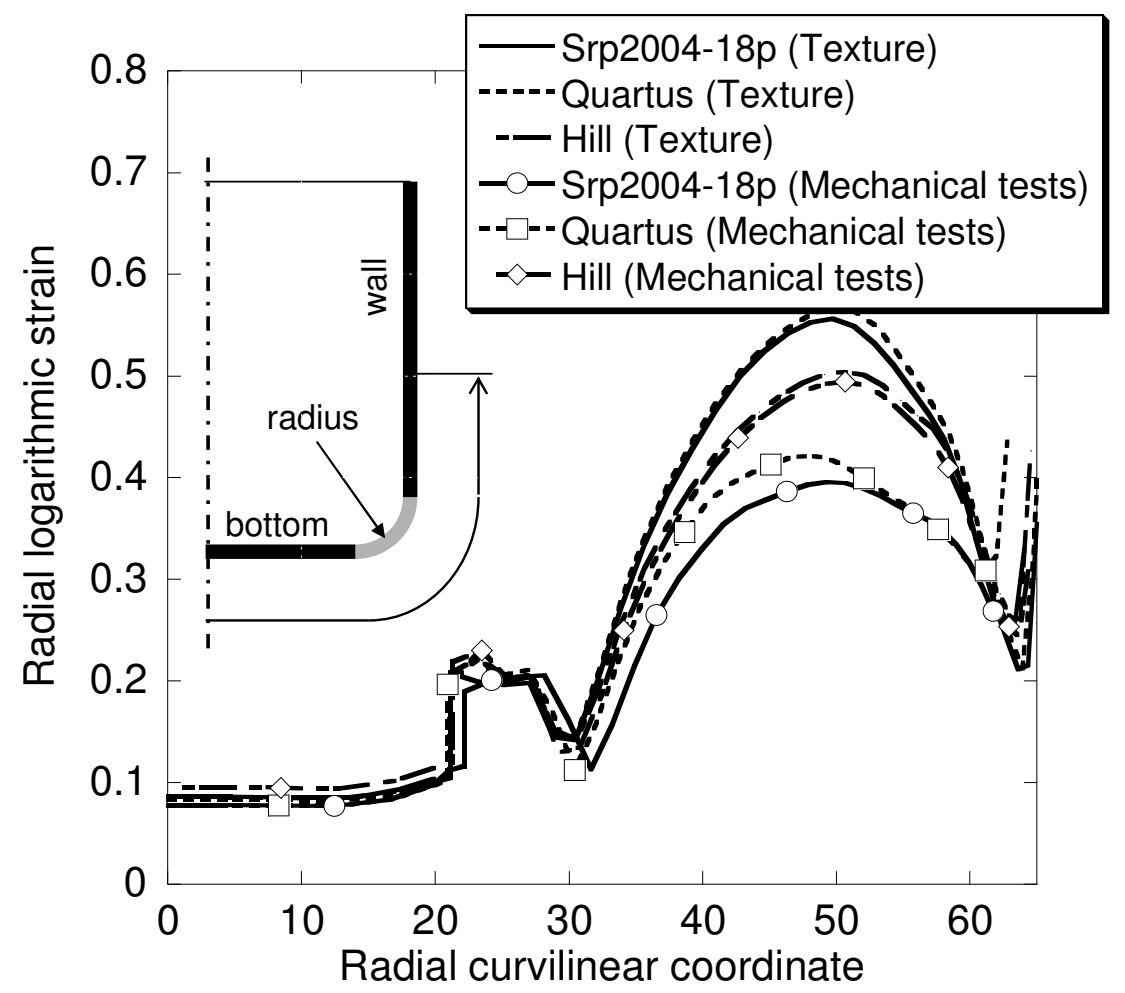

Figure 15. Impact of the strain-rate potential and identification procedure on the radial strain distribution predicted for AA6022.
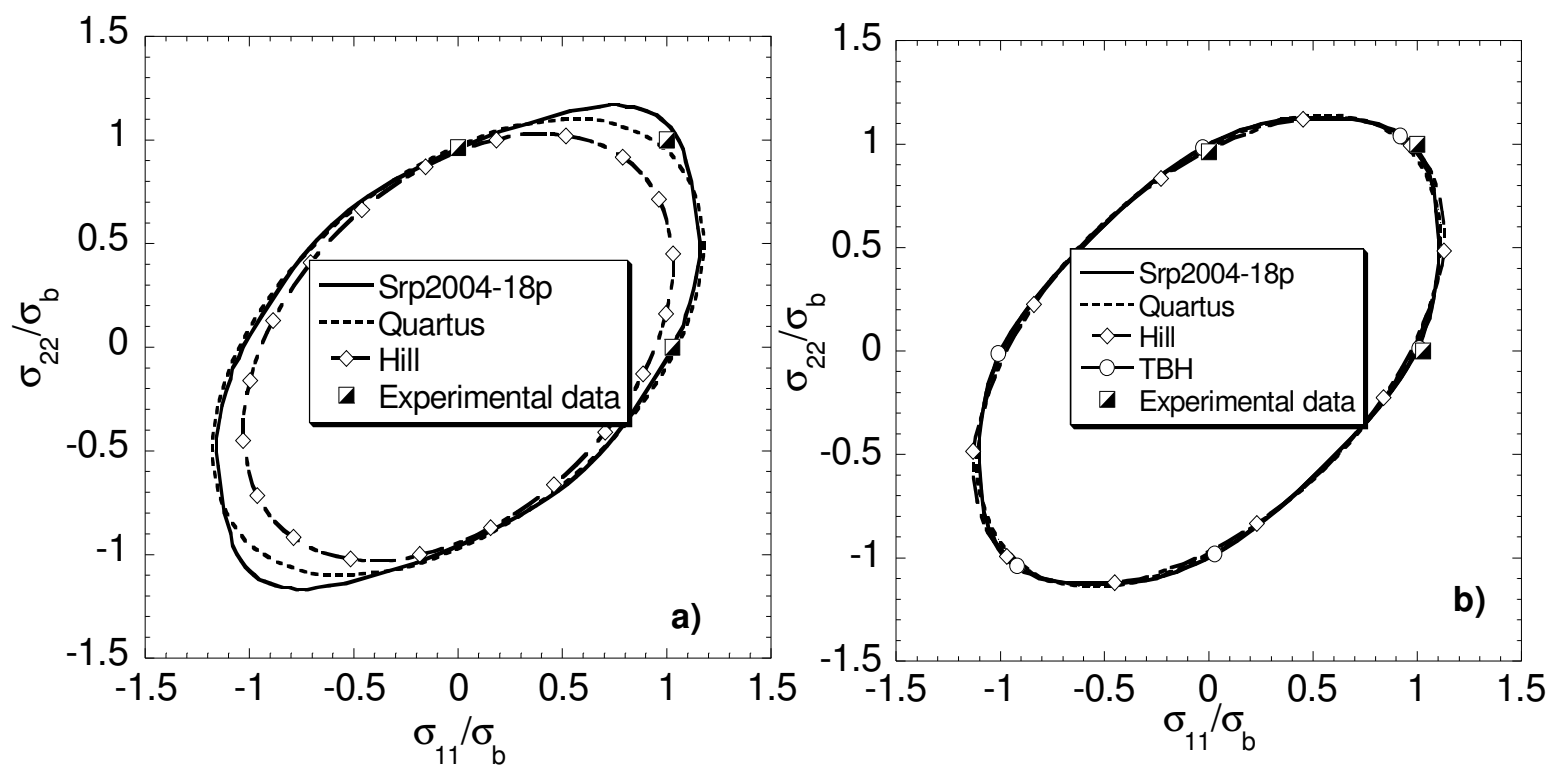

Figure 16. Predicted yield surfaces for AA6022 when the material parameters are identified, a) using the mechanical tests, and b) using the texture data and micromechanical simulations. 


\section{Final discussion and conclusions}

A series of different materials have been used for investigation of the finite element prediction of cup drawing tests by means of anisotropic strain-rate potentials, as a sequel to the parameter identification and analytical comparison performed in [34]. The recently proposed Srp2004-18p strain rate potential has been implemented in an elastic-plastic FE code and compared to the fourth-order Quartus potential and the quadratic Hill potential. The following conclusions can be drawn from the analysis of the results:

- Fairly coarse one-layer meshes of solid elements can be used for simulation of cup drawing, provided that reduced integration elements are avoided and that the stress distribution is not of interest;

- Technological and numerical parameters, e.g., pinching at the end of the holding phase, may strongly influence the cup rim profile, which is not the pure result of the material anisotropy;

- As a consequence, the analytical cup-height formula [11] predicts the correct number and location of the ears in all situations, yet the numerical simulation is required for a more quantitative evaluation of the cup height distribution.

- The quadratic potential is limited in describing both the $r$-values and the uniaxial yield stresses; however, its cup profile predictions were often acceptable for the materials and geometries analyzed in this paper;

- The advanced potentials, i.e., Quartus and Srp2004-18p, consistently predict the cup height distribution more accurately. This increase in accuracy is accompanied by a nonnegligible increase in computing time;

- The mathematical flexibility of Srp2004-18p resulted in the ability to predict up to ten ears in cup drawing; in particular, its predictions lay close to those of Yld2004-18p, therefore confirming their pseudo-duality;

- The automatic identification of advanced plastic potential parameters may sometimes lead to undesired results, especially when the number of experimental points is close or equal to the number of parameters. Even the number of ears may be predicted wrongly in some extreme situations. While the check-up of the $r$ and $\sigma$ graphs may be sufficient in most cases, it is suggested that the combined experiments-texture identification technique may be a safe yet automatic procedure that can be used to avoid such unrealistic results.

\section{Acknowledgements}

The authors are grateful to Frédéric Barlat for providing material data and to Cristian Teodosiu and Gérard Ferron for fruitful discussions and for reviewing early versions of this work. The first author is thankful to the Région Lorraine (France) for its three-year financial support.

\section{References}

[1] D. Banabic, H.-J. Bunge, K. Pöhlandt, A.E. Tekkaya, Formability of Metallic Materials, Springer-Verlag, 2000. 
[2] A.M. Habraken, Contributions to constitutive laws of metals: micro-macro and damage models, Thèse d'Agrégé de l'Enseignement Supérieur, Université de Liège, 2001 .

[3] K. Chung, M.G. Lee, D. Kim, C. Kim, M.L. Wenner, F. Barlat, International Journal of Plasticity. 21 (2005) 861-882.

[4] M.G. Lee, D. Kim, C. Kim, M.L. Wenner, K. Chung, International Journal of Plasticity. 21 (2005) 915-953.

[5] M.G. Lee, D. Kim, C. Kim, M.L. Wenner, R.H. Wagoner, K. Chung, International Journal of Plasticity. 21 (2005) 883-914.

[6] S. Bouvier, J.L. Alves, M.C. Oliveira, L.F. Menezes, Computational Material Science. 32 (2005) 301-315.

[7] B. Haddag, T. Balan, F. Abed-Meraim, International Journal of Plasticity. 23 (2007) 951-979.

[8] M. Gotoh, F. Ishise, Int. J. Mech. Sci. 20 (1978) 423.

[9] K. Chung, K. Shah, International Journal of Plasticity. 8 (1992) 453-476.

[10] A. Andersson, C.A. Ohlsson, K. Mattiason, B. Persson, in: J.C. Gelin, Picart, P. (Ed.), NUMISHEET'99, Vol. 1, Besancon, France, 1999, pp. 115-121.

[11] J.W. Yoon, F. Barlat, R.E. Dick, M.E. Karabin, International Journal of Plasticity. 22 (2006) 174-193.

[12] A.M. Habraken, L. Duchêne, International Journal of Plasticity. 21 (2004) 1525-1560.

[13] D. Raabe, F. Roters, International Journal of Plasticity. 21 (2004) 339-361.

[14] T. Böhlke, G. Risy, A. Bertram, Modelling and Simulation in Materials Science and Engineering. 14 (2006) 365-387.

[15] M. Arminjon, B. Bacroix, Acta Mechanica. 88 (1991) 219-243.

[16] M. Arminjon, B. Bacroix, D. Imbault, J.L. Raphanel, Acta Mechanica. 107 (1994) 3351.

[17] P. Van Houtte, K. Mols, A. Van Bael, E. Aernoudt, Textures Microstruct. 11 (1989) 23-39.

[18] F. Barlat, K. Chung, Modelling and Simulation in Materials Science and Engineering. 1 (1993) 403-416.

[19] K. Chung, F. Barlat, O. Richmond, J.W. Yoon, in: e.a. Zabaras (Ed.), The integration of Material, Process and Product Design, Balkema, Rotterdam, 1999, pp. 213-219.

[20] F. Barlat, K. Chung, in: D. Banabic (Ed.), 8th ESAFORM Conference, The Publishing House of the Romanian Academy, Cluj-Napoca, Romania, 2005, pp. 415-418. 
[21] D. Kim, F. Barlat, S. Bouvier, M. Rabahallah, T. Balan, K. Chung, International Journal of Plasticity. 23 (2007) 1380-1399.

[22] F. Barlat, K. Chung, O. Richmond, International Journal of Plasticity. 9 (1993) 51-63.

[23] F. Barlat, K. Chung, O. Richmond, Metallurgical and Materials Transactions A. 25 (1994) 1209-1216.

[24] K. Chung, S.Y. Lee, F. Barlat, Y.T. Keum, J.M. Park, International Journal of Plasticity. 12 (1996) 93-115.

[25] S.Y. Lee, Y.T. Keum, K. Chung, J.M. Park, F. Barlat, International Journal of Mechanical Sciences. 39 (1997) 1181-1198.

[26] K. Chung, F. Barlat, J.C. Brem, D.J. Lege, O. Richmond, International Journal of Mechanical Sciences. 39 (1997) 105-120.

[27] J.W. Yoon, I.S. Song, D.Y. Yang, K. Chung, F. Barlat, International Journal of Mechanical Sciences. 37 (1995) 733-752.

[28] P. Van Houtte, A. Van Bael, J. Winters, Textures and Microstructures. 24 (1995) 255272.

[29] B. Bacroix, P. Gilormini, Modelling and Simulation in Materials Science and Engineering. 3 (1995) 1-21.

[30] Y. Zhou, J.J. Jonas, J. Savoie, A. Makinde, S.R. MacEwen, International Journal of Plasticity. 14 (1998) 117-138.

[31] S. Li, E. Hoferlin, A. Van Bael, P. Van Houtte, C. Teodosiu, International Journal of Plasticity. 19 (2003) 647-674.

[32] J.H. Kim, M.G. Lee, F. Barlat, R.H. Wagoner, K. Chung, International Journal of Plasticity. 24 (2008) 2298-2334.

[33] M. Rabahallah, T. Balan, S. Bouvier, C. Teodosiu, Int. J. Num. Meth. Engineering. (2009) accepted.

[34] M. Rabahallah, T. Balan, S. Bouvier, B. Bacroix, F. Barlat, K. Chung, C. Teodosiu, International Journal of Plasticity. 25 (2009) 491-512.

[35] P. Van Houtte, S.K. Yerra, A. Van Bael, International Journal of Plasticity. (2008) doi: 10.1016/j.ijplas.2008.1002.1001.

[36] M. Rabahallah, T. Balan, F. Barlat, International Journal of Solids and Structures. 46 (2009) 1966-1974.

[37] L. Bourne, R. Hill, Phil. Mag. 41 (1950) 49-70.

[38] W.F. Hosford, R.M. Caddell, Metal Forming: Mechanics and Metallurgy, PrenticeHall, Englewood Cliffs, NJ, 1983.

[39] F. Barlat, S. Panchanadeeswaran, O. Richmond, Metall. Trans. A. 22 (1991) 1525. 
[40] H. Ziegler, An introduction to thermomechanics, North-Holland, Amsterdam, 1977.

[41] R. Hill, Journal of Mechanics and Physics of Solids. 35 (1987) 22-33.

[42] M. Gotoh, Int. J. Mech. Sci. 19 (1977) 505-520.

[43] J.W. Yoon, D.Y. Yang, K. Chung, Computer Methods in Applied Mechanics and Engineering. 174 (1999) 23-56.

[44] M. Rabahallah, Modélisation de l'anisotropie plastique et application à la mise en forme des tôles métalliques, PhD Thesis, University of Metz, 2007.

[45] P. Van Houtte, A. Van Bael, International Journal of Plasticity. 20 (2004) 1505-1524.

[46] K. Chung, O. Richmond, International Journal of Plasticity. 9 (1993) 907-920.

[47] J.E. Dennis, R.B. Schnabel, Numerical methods for unconstrained optimization and non-linear equations, Prentice Hall, Englewood Cliffs, NJ, USA, 1983.

[48] S. Li, E. Hoferlin, A. Van Bael, P. Van Houtte, Advanced Engineering Materials. 3 (2001) 990-994.

[49] L.P. Moreira, Étude numérique de l'influence du modèle de plasticité sur le comportement des tôles lors de l'emboutissage, PhD Thesis, Université de Metz, 2002.

[50] L.P. Moreira, G. Ferron, Journal of Materials Processing Technology. 155-156 (2004) 1596-1603.

[51] L. Duchêne, P. De Montleau, F. El Houdaigui, S. Bouvier, A.M. Habraken, in: L.M. Smith, F. Pourboghat, J.W. Yoon, T.B. Stoughton (Eds.), Numisheet 2005, 6th International Conference and Workshop on Numerical Simulations of 3D Sheet Forming Processes, Detroit, Michigan, USA, 2005.

[52] S. Bouvier, J.L. Alves, A.M. Habraken, C. Teodosiu, International Journal of Plasticity. (submitted).

[53] S. Bouvier, C. Teodosiu, C. Maier, M. Banu, V. Tabacaru, 2001.

[54] H. Haddadi, S. Bouvier, M. Banu, C. Maier, C. Teodosiu, International Journal of Plasticity. 22 (2006) 2226-2271.

[55] J.L. Alves, Simulação numérica do processo de estampagem de chapas metálicas: Modelação mecânica e métodos numéricos, University of Minho, 2003.

[56] S. Bouvier, L. Alves, A.M. Habraken, AIP Conference Proceedings, Vol. 907, Zaragoza, 2007, pp. 94-99.

[57] F. Grytten, B. Holmedal, O.S. Hopperstad, T. Børvik, International Journal of Plasticity. 24 (2008) 2248-2277.

[58] T. Balan, B. Bacroix, C. Teodosiu, Proc. TPR 2000, Cluj-Napoca, Romania, 2000, pp. 81-93. 
